\title{
Relativistic emission lines from accreting black holes
}

\section{The effect of disk truncation on line profiles}

\begin{abstract}
A. Müller and M. Camenzind
Landessternwarte Koenigstuhl, 69117 Heidelberg, Germany

Received 30 July 2003 / Accepted 22 September 2003

Abstract. Relativistic emission lines generated by thin accretion disks around rotating black holes are an important diagnostic tool for testing gravity near the horizon. The iron $\mathrm{K}$-line is of special importance for the interpretation of the X-ray emission of Seyfert galaxies, quasars and galactic X-ray binary systems. A generalized kinematic model is presented which includes radial drifts and non-Keplerian rotations for the line emitters. The resulting line profiles are obtained with an object-oriented ray tracer operating in the curved Kerr background metric. The general form of the Doppler factor is presented which includes all kinds of poloidal and toroidal motions near the horizon. The parameters of the model include the spin parameter, the inclination, the truncation and outer radius of the disk, velocity profiles for rotation and radial drift, the emissivity profile and a multi-species line-system.

The red wing flux is generally reduced when radial drift is included as compared to the pure Keplerian velocity field. All resulting emission line profiles can be classified as triangular, double-horned, double-peaked, bumpy and shoulder-like. Of particular interest are emission line profiles generated by truncated standard accretion disks (TSD). It is also shown that the emissivity law has a great influence on the profiles. The characteristic shoulder-like line profile observed for the Seyfert galaxy MCG-6-30-15 can be reproduced for suitable parameters.
\end{abstract}

Key words. galaxies: active - galaxies: Seyfert - accretion - relativity - black hole physics - line: profiles

\section{Introduction}

Emission lines originating from regions where the influence of curved space-time can not be neglected are found in accreting black hole systems like Active Galactic Nuclei (AGN), microquasars and Galactic Black Hole Candidates (GBHC). These astrophysical systems exhibit hot plasma falling into the deep potential well of black holes. The ion species in the plasma still have some electrons on lower shells so that there are several transitions between electron states that generate emission lines in the $\mathrm{X}$-ray range. The most prominent line is the $\mathrm{Fe} \mathrm{K} \alpha$ line at $6.4 \mathrm{keV}$ rest frame energy (nearneutral iron). Additionally, the $\mathrm{Fe} \mathrm{K} \beta$ line at $7.06 \mathrm{keV}$ and rarely Ni $\mathrm{K} \alpha$ at $7.48 \mathrm{keV}$ and $\mathrm{Cr} \mathrm{K} \alpha$ at $5.41 \mathrm{keV}$ can be applied to fit X-ray emission line systems. The fluorescence process and its transition energies depend on the ionization state of the species. One can distinguish hot and cold regimes of line emission which can be described by an ionization parameter (Ballantyne et al. 2001). Generally, X-ray astronomers observe a multi-component emission line complex of these species. The Fe $\mathrm{K} \alpha$ line dominates the line system due to its high relative strength and is often indicated in unreduced global X-ray spectra.

Send offprint requests to: A. Müller,

e-mail: amueller@lsw.uni-heidelberg.de
Broad X-ray emission lines originate from the hot inner parts of accreting black hole systems, typically from radii comparable to the radius of marginal stability. Naturally, these lines hint for a proximity of hot and cold material: hot material can be found in an optically thin corona, a place where cold seed photons are Comptonized to hard radiation. It is expected that the corona becomes optically thick at super-Eddington accretion rates and is dominated by Comptonization. This latter scenario seems to hold for narrow-line Seyfert-1 galaxies (NLS1s), whereas classical broad-line Seyfert-1 galaxies (BLS1s) accrete at sub-Eddington to Eddington rates.

Relatively cold material hides in the radiatively-efficient, geometrically thin and optically thick accretion disk, the so called standard disk, hereafter SSD (Shakura-Sunyaev Disk) (Shakura \& Sunyaev 1973). The resulting structure equations for this standard disk have been generalized to the relativistic case later (Novikov \& Thorne 1974). The hard coronal input radiation usually described by a power law illuminates the cold disk. As a consequence, the surface of the disk is ionized in a thin layer. In a fluorescence mechanism the hard radiative input is absorbed (threshold at $7.1 \mathrm{keV}$ ) and re-emitted in softer fluorescence photons. But in the dominant competitive process, the auger effect, the excited ions emit auger electrons and enrich the environment with hot electrons. One of the first applications of this X-ray fluorescence was performed at the microquasar 
Cyg X-1 (Fabian et al. 1989). These observations were supplemented by radio-quiet AGN, e.g. Seyfert galaxies, showing the same X-ray reflection signatures (Pounds et al. 1990; Tanaka et al. 1995; Reynolds \& Nowak 2003).

But there are also sources exhibiting narrow $\mathrm{X}$-ray emission lines. The narrow lines do not show distortion by relativistic effects. It has been proposed that narrow $\mathrm{X}$-ray emission lines can be explained by reflection at the large-scale dusty torus (Turner et al. 1997). Here, the narrow line is overimposed on broad line profiles. This interpretation has been applied to some sources, like Seyfert galaxies, e.g. MCG-6-30-15 (Wilms et al. 2001), MCG-5-23-16 (Dewangan et al. 2003) and Quasars, e.g. Mrk 205 (Reeves et al. 2001).

The absence of the line feature in several Sefert-1 galaxies (Pfefferkorn et al. 2001) can be explained by global accretion models. The first possible interpretation is that the inner edge of the disk seems to be far away from the black hole. If the fluorescence process is still possible in that distance, the skewness of the X-ray emission line vanishes and becomes rather Newtonian-like. In even larger distances, it is supposed that the emission line feature disappears completely because irradiation and fluorescence is reduced. Accretion theory states that this scenario holds for low accretion rate: then the transition radius between standard accretion disk and optically thin disk is large (Esin et al. 1997). The mass determinations for Seyfert black holes confirm this interpretation while being in the intermediate range of $10^{6}$ to $10^{8} M_{\odot}$. These lighter supermassive black holes seem to accrete very weakly.

$\mathrm{X}$-ray spectra of AGN and X-ray binaries are dominated by broad Comptonized continua. The properties of these spectra are discussed in the next section. The first step for astronomers is to reduce the observed data files and to extract the line profile by subtracting this global continuum emission. This procedure is already complicated because the intrinsic curvature of the Comptonized radiation is still topic of an ongoing debate.

The resulting emission line profile serves for diagnostics and to constrain essential parameters of the accreting black hole system. So, astronomers found an X-ray imprint of black holes and can derive properties concerning the relative position from the inner accretion disk (standard disk) to the observer, the inclination $i$ or the rotational state of the black hole, the Kerr rotational parameter $a$, the extension of the geometrically thin and optically thick standard disk, like inner radius $r_{\text {in }}$ and outer radius $r_{\text {out }}$, the velocity field of the plasma, separated in toroidal $v^{\Phi}$, poloidal $v^{\Theta}$ and radial $v^{\mathrm{r}}$ components and the relative position of hot corona to cold accretion disk.

Let us follow up the theoretical steps from black hole theory needed here until one gets simulated emission line profiles: Sect. 3 introduces the metric of rotating black holes, the Kerr geometry, and provides the standard co-ordinate system in Boyer-Lindquist form. Then, the generalized GR Doppler factor is presented. The derivation of this quantity and its signification in GR ray tracing is elaborated, too.

In Sect. 4, realistic plasma kinematics resulting from hydrodynamic and magnetohydrodynamic accretion theory is discussed. The Kerr geometry has special features that are valid

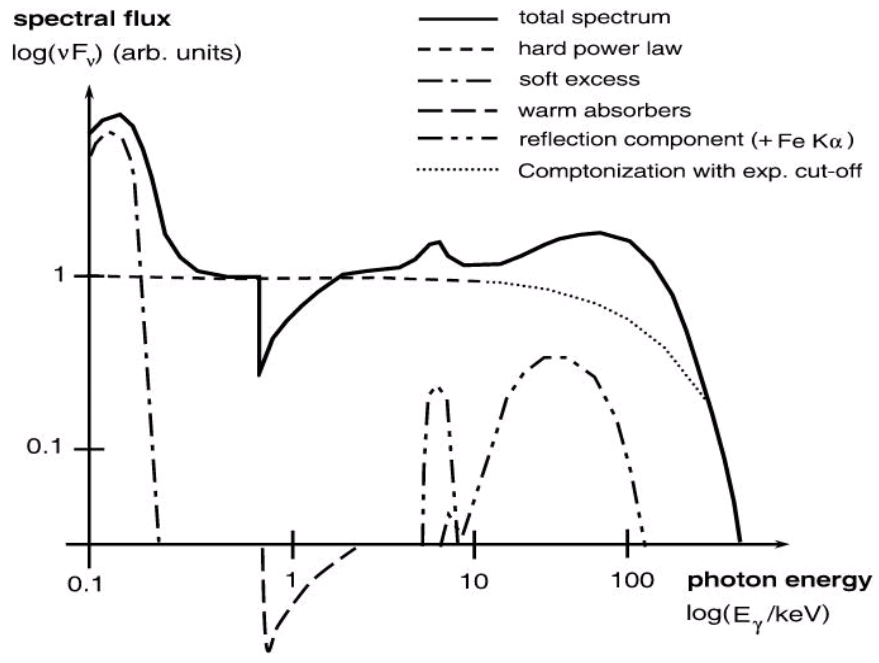

Fig. 1. Prototype X-ray spectrum of a type-1 AGN (illustration idea taken from Fabian 1998). The spectrum is dominated by the global Comptonized continuum. At low energies, there is the soft excess, in principle a composition of some black bodies belonging to the disk divided into rings. At energies around $1 \mathrm{keV}$, one can recognize a complex of absorption dips, originating from the warm absorbers. The reflection component consists of a broad bump peaking around $20 \mathrm{keV}$ and the prominent fluorescence emission line complex around $6.4 \mathrm{keV}$.

independently from the accretion model! This is accessable by analytical investigations.

Another basic ingredient to calculate emission lines and spectra in general is the emissivity law that determines the emission behaviour of the line emitting region. In Sect. 5, alternative emissivity shapes besides th classical single power law are applied.

Ray racing provides in a first step disk images. It may be possible to resolve these images observationally in near future. In any case, disk images as presented in Sect. 6 are very instructive unrevealing GR effects and influence of plasma dynamics.

In Sect. 7, the calculation and basic properties of the emission lines are discussed.

In the final Sect. 8, the parameter space of relativistic emission lines is investigated and suitable criteria to fix line features are presented. One can apply these criteria to observed line profiles and derive a classification in the line jungle.

\section{X-ray spectra and topology of type-1 AGN}

The data quality increased conspicuously with the launch of the space-based X-ray observatories Chandra and XMM-Newton. The challenge in interpreting X-ray spectra of AGN and XRBs is to correctly reduce the data and subtract the continuum. Afterwards one can discuss the formation of other spectral components, such as emission lines.

$\mathrm{X}$-ray spectra of Seyfert galaxies have been investigated for several years. These types of AGN were the first objects that gave evidence for rotating black holes (Tanaka et al. 1995) and confirmed the paradigm of the AGN engine: an accreting supermassive black hole producing high multi-wavelength luminosity. Only a few years later, high-resolution observatories 
allowed the extraction of iron $\mathrm{K}$ emission lines in more distant objects, Quasars (Yaqoob et al. 1999). In the course of the unification scheme of AGN, the different AGN types are considered as different evolution phases of a prototype AGN with some pecularities that mainly arise by differencies in orientation of the accretion disk, accretion rate and black hole mass. Especially, the discrimination between Seyferts and Quasars has become somewhat arbitrary. Only the amount of radiation, $\approx 10^{11} L_{\odot}$ for Seyferts and $10^{13}$ to $10^{15} L_{\odot}$ for Quasars, is the main difference between these two subclasses. Concerning the iron emission line feature, it can be stated that Quasars exhibit increased ionization of the standard disk surface due to enhanced central luminosity. Therefore, weaker emission line signatures are expected (Reynolds \& Nowak 2003).

The dichotomy phenomenon caused by disk orientation and dust torus obscuring, a generalization that can be applied to Seyferts and Quasars, is well-known: one can distinguish type-1 (low inclination) and type-2 AGN (high inclination). Hence, it is for observational reasons only possible to study relativistic emission lines of AGN of type 1 .

$\mathrm{X}$-ray spectra of Seyfert galaxies in the range from 0.1 to several $100 \mathrm{keV}$ show many features as illustrated in Fig. 1: overlaying the complete $\mathrm{X}$-ray range there is a continuum originating from Comptonized radiation. This is the direct radiation that reaches the observer coming from a hot corona $(T \approx 30 \mathrm{keV})$. The photon number flux per unit energy is usually modelled with a power law distribution, $F_{\mathrm{N}}(E) \propto$ $E^{-\Gamma}$. The soft input radiation from the cold accretion disk is reprocessed via unsaturated inverse Compton scattering by ultra-relativistic electrons in the corona. Figure 2 depicts in a simple way one probable geometry of an accreting black hole systems that hold especially for AGN and microquasars, known as "sphere+disk geometry". Matter has angular momentum and therefore spirales down in a flat and cold standard disk. The argument for the flatness is because of vertical collapse due to efficient radiation cooling. The relative scale heigth $H / r$ of these standard disks is only $10^{-3}$ (Shakura \& Sunyaev 1973). The matter within this cold disk emits a multi-color blackbody spectrum. This is simply the superposition of a sequence of Planck spectra, e.g. rings each with a temperature $T_{\mathrm{i}}$. The innermost ring is the hottest one, $T_{\text {in }} \geq T_{\mathrm{i}} \geq T_{\text {out }}$. The accretion rate determines the transition radius and this one fixes the temperature of the inner edge of the disk. At high accretion rates, the inner edge is significantly hot so that a soft excess can be observed around $1 \mathrm{keV}$. At small accretion rates the soft excess lacks (Esin et al. 1997). The cold and thin accretion disk delivers the soft seed photons that are Comptonized in the hot, optically thin corona. The topology of the corona is still one of the essential open questions in X-ray spectroscopy. Different assumptions such as slab and patchy corona models have been made (Reynolds \& Nowak 2003). The observed hard spectra suggest rather "sphere+disk geometries" because slab and patchy corona models are more efficiently radiative-cooled by seed photons from the cold disk. The reverberation mapping technique may enlighten the spatial position of corona to accretion disk. Theoretically, it will be task of upcoming relativistic MHD simulations to unreveal the corona-disk geometry depending on accretion rate and radiative cooling. Apparently, it

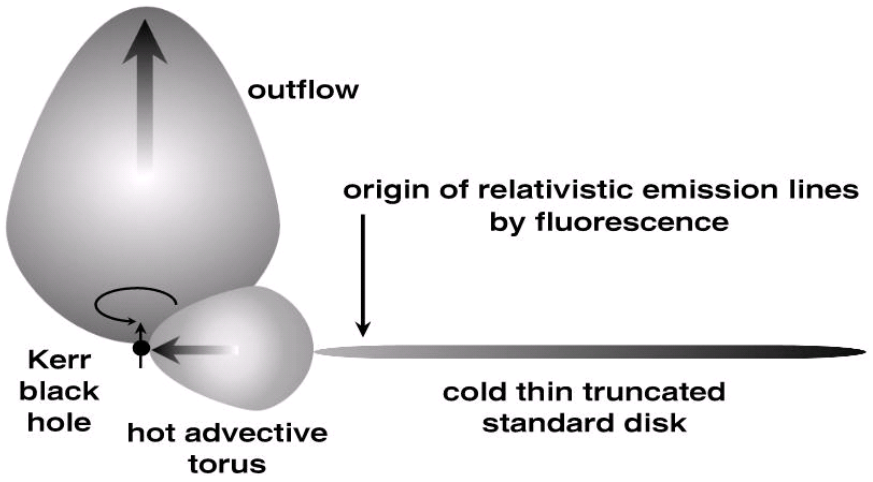

Fig. 2. Illustration of topological elements in the innermost region of accreting black hole systems. The size and morphology of these elements depend on accretion rate, black hole mass and radiative cooling.

turns out that the coronal emissivity is significantly enhanced at the inner edge of the standard disk (Merloni \& Fabian 2003) and that MHD dissipation plays a crucial role to invalidate the zero-torque boundary condition. Then, magnetically-induced torques at radii comparable to the radius of marginal stability are expected to increase dissipation. This mechanism may provide the higher disk emissivities $\epsilon(r) \propto r^{-4.5}$ as recently observed in Seyfert galaxy MCG-6-30-15 (Wilms et al. 2001).

Here it is assumed that the corona is almost identical with an advection-dominated torus that forms by feeding from the standard disk. An alternative may be the quasi-spherical rather Bondi-accreting Advection-Dominated Accretion Flow (ADAF) (Narayan \& Yi 1994) that forms under other circumstances in accretion theory, e.g. depending on the accretion rate. The transition region where the standard disk adjoins the hot torus is unstable. Hot corona and cold disk may constitute a sandwich configuration where the inner edge of the cold disk oscillates in radial direction (Gracia 2003). This ADAFSSD transition region seems to depend on the efficiency of different cooling channels as can be found in hydrodynamic simulations (Manmoto \& Kato 2000; Gracia 2002). More recent pseudo-Newtonian hydrodynamic simulations incorporating radiation cooling via synchrotron radiation, bremsstrahlung and Comptonization with the consideration of conduction in a two-component plasma suggest disk truncation (Hujeirat \& Camenzind 2000). This means that the accretion disk can cut off even at radii larger than the radius of marginal stability, $r_{\mathrm{ms}}$. Truncation of standard disks (TSD) in general softens the argument for observed evidences of rapidly spinning black holes. This is because truncated disks do not extend to $r_{\mathrm{ms}}$ inwardly. However the decreasing $r_{\mathrm{ms}}$ for faster spinning black holes was always the argument for evidence of Kerr black holes (Iwasawa et al. 1996).

Particularly, the cooling time-scale of synchrotron radiation is typically in the millisecond domain and cools very fast the hot accretion flow. The Truncated Disk - Advective Torus (TDAT) scenario (Hujeirat \& Camenzind 2000) produces a hot inner torus that is not stable either. It is destablized by accretion and matter free-falls into the black hole. But a small fraction of matter can escape the black hole and is transported along 


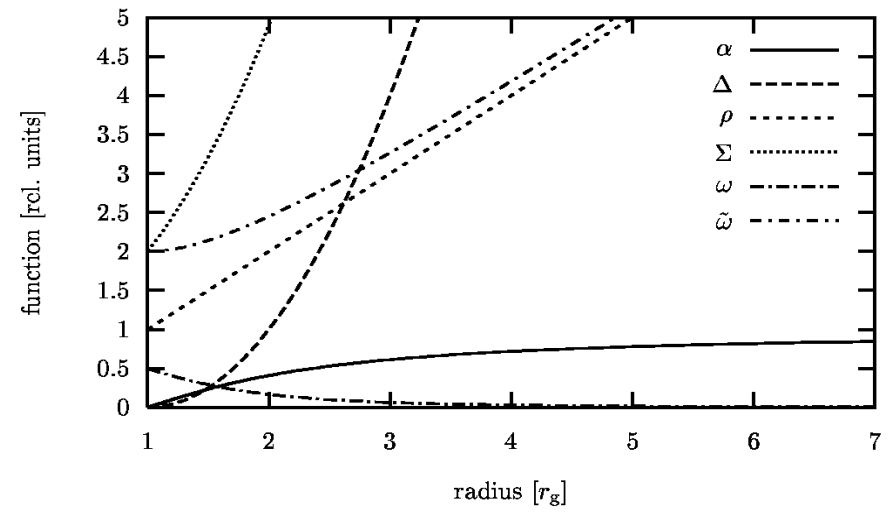

Fig. 3. Radial behaviour of the Boyer-Lindquist functions at extreme Kerr $(a=1.0)$ in the equatorial plane. Relativistic units were used.

magnetic field lines in polar regions. This drives outflows that consist of disk winds on one hand and of ergospheric Poynting fluxes on the other hand. This outflow is supposed to feed the large-scale jets of AGN or the blobs of microquasars. In a unified view, the total accretor mass, $M$, and the total mass accretion rate, $\dot{M}$, are strongly favoured to determine the spectral state and the jet injection rate and the continuity of the outflow. Relativistic emission lines may serve as a diagnostic tool to fix these parameters.

The continuum of the hot corona reveals an intrinsical curvature and cuts off at the high-energy branch at several hundreds of $\mathrm{keV}$. The cut-off at $3 k_{\mathrm{B}} T_{\mathrm{C}}$ indicates the plasma temperature in the corona (Rybicki \& Lightman 1979). At the low energy branch, some sources show a soft excess with a bumpy profile. This can be identified with a multi-color blackbody originating from the cold Compton-thick accretion disk. At $1.0 \mathrm{keV}$ there is a complex structure of warm absorbers, typically a combination of adjacent absorption edges of a variety of species (C V, O VII, O VIII, Ne IX etc.). These dips and their potentially relativistic broadening are still topic of an ongoing debate (Mason et al. 2003; Lee 2001). The reflection component consists of a system of emission lines, most prominently the iron $\mathrm{K} \alpha$ at $6.4 \mathrm{keV}$ typically, and a broad bump peaking at 20 to $30 \mathrm{keV}$. The reflection bump is a consequence of hard radiation from the corona hitting the cold accretion disk that is then reflected to the observer (Reynolds 1996). The origin of the iron $\mathrm{K} \alpha$ line is well understood: at typical temperatures of $10^{5}$ to $10^{7} \mathrm{keV}$ in the inner accretion disk, iron is ionized but not completely stripped; the $\mathrm{K}$ - and $\mathrm{L}-$ shell are still populated. First, iron is excitied by photo-electric absorption. The threshold lies at $\approx 7.1 \mathrm{keV}$ so that only hard $\mathrm{X}$-ray photons from the corona can release this primary process. Then, there are two competing processes: the dominant process ( $66 \%$ probability) is the Auger effect where the excitation energy is emitted with an Auger electron. This mechanism is non-radiative and enriches the disk plasma with electrons. The second and here essential process is fluorescence (34\% probability): one electron of the L-shell undergoes the transition to the $\mathrm{K}$-shell accompanied by the emission of the iron $\mathrm{K} \alpha$ fluorescence photon with $6.4 \mathrm{keV}$ rest frame energy ${ }^{1}$. The emission line energy and the

${ }^{1}$ We neglect here any distinction of the $\mathrm{K} \alpha_{1}$ line at $6.404 \mathrm{keV}$ and $\mathrm{K} \alpha_{2}$ line at $6.391 \mathrm{keV}$ as can be found in atomic physics. existence of the fluorescence line in general depend on the ionization state of the material (Ross et al. 1999).

The significance of other elements contributing to the reflection component is rather low, because the abundance, relative strength and fluorescence yield is low compared to that of iron (Reynolds 1996). At most, the iron $\mathrm{K} \beta$ at $7.06 \mathrm{keV}$ or nickel at $7.48 \mathrm{keV}$ (Wang et al. 1999) and chromium at $5.41 \mathrm{keV}$ could contribute marginally. Besides, the accretion disk has only a thin ionization layer. The line producing layer has only the depth of 0.1 to $1 \%$ of the disk thickness (Matt et al. 1997). Therefore the contributions of ionized iron from deeper regions - or even a radiation transfer problem from lower ionized slabs - can be neglected due to high optical depths.

\section{Ray tracing in the Kerr geometry}

The Kerr metric describes rotating uncharged black holes. This vacuum solution of the Einstein field equations (Kerr 1963) generalizes the static Schwarzschild solution. The line element fullfills the standard form of stationary and axisymmetric space-times

$\mathrm{d} s^{2}=e^{2 \Phi} \mathrm{d} t^{2}-e^{2 \Psi}(\mathrm{d} \Phi-\omega \mathrm{d} t)^{2}-e^{2 \mu_{\mathrm{r}}} \mathrm{d} r^{2}-e^{2 \mu_{\Theta}} \mathrm{d} \Theta^{2}$.

The Kerr geometry in Boyer-Lindquist form is determined by the line element

$\mathrm{d} s^{2}=-\alpha^{2} \mathrm{~d} t^{2}+\tilde{\omega}^{2}(\mathrm{~d} \Phi-\omega \mathrm{d} t)^{2}+\left(\rho^{2} / \Delta\right) \mathrm{d} r^{2}+\rho^{2} \mathrm{~d} \Theta^{2}$,

with the canonical Boyer-Lindquist functions

$\alpha=\frac{\rho \sqrt{\Delta}}{\Sigma}$,

$\Delta=r^{2}-2 M r+a^{2}$,

$\rho^{2}=r^{2}+a^{2} \cos ^{2} \theta$,

$\Sigma^{2}=\left(r^{2}+a^{2}\right)^{2}-a^{2} \Delta \sin ^{2} \theta$,

$\tilde{\omega}=\frac{\Sigma}{\rho} \sin \theta$,

$\omega=\frac{2 a M r}{\Sigma^{2}}$

$M$ is the mass and $a$ the spin parameter of the black hole.

The Boyer-Lindquist co-ordinates are pseudo-spherical. The expressions above are called frame-dragging frequency or potential for angular momentum $\omega$, cylindrical radius $\tilde{\omega}$, lapse function or redshift factor $\alpha$ and some geometrical functions $\Delta$ and $\rho$.

The rotation of the black hole can be parametrized by its specific angular momentum $a$, the so called Kerr parameter. Relativistic units $G=M=c=1$ are used unless otherwise stated. Then, the natural length-scale is the gravitational radius, $r_{\mathrm{g}}=G M / c^{2}$, and $a$ varies between -1 and 1 . The interval $a \in[-1,0]$ involves retrograde rotation between black hole and disk, the interval $a \in[0,1]$ means prograde rotation. From black hole theory, it is generally possible that $a$ takes all values in this interval, but accretion theory suggests that there is mainly one type of black holes: rotating Kerr black holes near its maximum rotation $(a \approx 1)$. This is because black holes of mass $M$ are spun up to $a \simeq \Delta m / M$ by accreting a mass $\Delta m$. At least the supermassive black holes in AGN 
that accrete for long times are expected to rotate very rapidly, $a \geq 0.9$. It is possible to extract angular momentum from black holes via Blandford-Znajek (Blandford \& Znajek 2000) and Penrose processes (Penrose \& Floyd 1971). But these effects may not be efficient enough to slow down the black hole rotation significantly.

Figure 3 illustrates the radial dependence of the BoyerLindquist functions in the equatorial plane $(\theta=\pi / 2)$ at the maximum value of black hole rotation, $a=1$. One can see the frame-dragging frequency $\omega$ that increases significantly at smaller radii and reaches $\omega\left(r_{\mathrm{H}}\right)=\Omega_{\mathrm{H}}=1 / 2$ in relativistic units at the horizon. This behaviour shows that dragging of inertial frames is important when reaching the ergosphere. Another essential feature is the steep decrease of the redshift factor $\alpha$. This is mainly the cause that emission is strongly suppressed when the distance becomes smaller than approximatly the radius of marginal stability, $r_{\mathrm{ms}}$, which satisfies

$r_{\mathrm{ms}}=M\left(3+Z_{2} \mp \sqrt{\left(3-Z_{1}\right)\left(3+Z_{1}+2 Z_{2}\right)}\right)$,

$Z_{1}=1+\left(1-\frac{a^{2}}{M^{2}}\right)^{1 / 3}\left(\left(1+\frac{a}{M}\right)^{1 / 3}+\left(1-\frac{a}{M}\right)^{1 / 3}\right)$

$Z_{2}=\sqrt{3 \frac{a^{2}}{M^{2}}+Z_{1}^{2}}$,

(upper sign prograde, lower sign retrograde orbits).

Our object-oriented code KBHRT (Kerr Black Hole Ray Tracer) (Müller 2000) programmed in $\mathrm{C}++$ is used to derive in the first step the image of a relativistic disk and in the second step to calculate the line flux by integrating over this image and weighting with the relativistic generalized Doppler factor and the radial disk emissivity profile. The imaging of the disk is not observationally possible because one can not (yet) resolve these distant accreting black hole systems. But these images show nicely the influence of curved space-time on photon paths. The flux is indeed the observationally accessible information and can be determined afterwards.

The code which is based on a C-code (Fanton et al. 1997) calculates the photon path by integrating the GR geodesics equation using the complete set of integrals of motion. Besides the canonical conservative quantities like mass, energy and angular momentum, the Kerr geometry exhibits a fourth one: the Carter constant (Carter 1968). This quantity is associated with the radial and poloidal photon momentum. The second order null geodesics equation reduces by means of these four conservatives to a set of four first order ordinary differential equations which can easily be integrated via elliptical integrals or Runge-Kutta schemes. The code is very fast because the position on the disk $(r, \theta, \Phi)$ is folded directly onto the observers screen $(x, y)$ without following the path stepwise between these two 2D objects (see Fig. 4). This is an alternative to the first historical method using transfer functions (Cunningham 1975) which is still in use (Bromley et al. 1997; Speith et al. 1995) or direct shooting, a method that follows the complete ray from locus of emission to observer. The constraint of dealing only with flat disks that lay infinitely thin in the equatorial plane is a good approximation to the vertical collapsed geometrically thin standard disks, however it is a restriction of our code. One recent issue shows the direction of future work, where the ray tracing

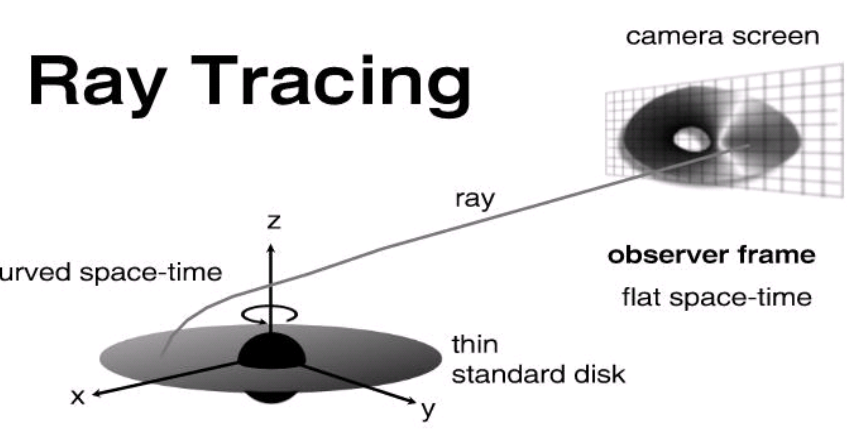

black hole - disk system

Fig. 4. Schematical representation of a Kerr ray tracer. The light rays start at the screen (back tracking) and hit the disk surface in the equatorial plane. On the screen the lensed image is formed as seen by a distant observer. Our solver folds directly from equatorial plane to screen.

code has to be coupled to hydrodynamical or MHD accretion models (Armitage \& Reynolds 2003). This coupling allows to study the variable emission near black holes.

The KBHRT code encapsulates all relevant tools to visualize relativistic disks and emission lines. One can study the distribution of the generalized Doppler factor allover the disk or the emission itself.

The generalized Doppler factor is defined by

$g \equiv \frac{v_{\mathrm{obs}}}{v_{\mathrm{em}}}=\frac{1}{1+z}=\frac{\hat{p}_{\mathrm{obs}}^{\mathrm{t}}}{\hat{p}_{\mathrm{em}}^{\mathrm{t}}}$,

where $v_{\mathrm{obs}}$ and $v_{\mathrm{em}}$ correspond to observed and emitted photon momenta, $z$ to the redshift and $\hat{p}$ denotes the rest frame of the plasma. The Zero Angular Momentum Observer (ZAMO) or Bardeen observer momenta have to be boosted into the rest frame of the plasma to derive $g$ by means of a local Lorentz transformation,

$\hat{p}^{\mathrm{t}}=\gamma\left[p^{(\mathrm{t})}-v^{(\mathrm{j})} p_{(\mathrm{j})}\right]$,

$\hat{p^{\mathrm{i}}}=p^{(\mathrm{i})}+\frac{1}{v^{2}}\left[(\gamma-1) p_{(\mathrm{j})} v^{(\mathrm{j})} v^{(\mathrm{i})}-\gamma p^{(\mathrm{t})} v^{(\mathrm{i})}\right]$,

with the Lorentz factor $\gamma$ as measured by ZAMOs, the Carter momenta $p^{(\mu)}$ and the modulus of the velocity $v$. Only the component $\hat{p}^{\mathrm{t}}$ is needed and one gets

$$
\begin{aligned}
\hat{p}^{\mathrm{t}} & =\gamma\left[p^{(\mathrm{t})}-v^{(\mathrm{r})} p_{(\mathrm{r})}-v^{(\theta)} p_{(\theta)}-v^{(\Phi)} p_{(\Phi)}\right] \\
& =\gamma\left[\frac{1}{\alpha}(1-\omega \lambda)-v^{(\mathrm{r})} \frac{\sqrt{R}}{\rho \sqrt{\Delta}}-v^{(\theta)} \frac{\sqrt{\Theta}}{\rho}-v^{(\Phi)} \frac{\lambda}{\tilde{\omega}}\right]
\end{aligned}
$$

With $\hat{p}_{\text {obs }}^{\mathrm{t}} \rightarrow 1$ for $r \rightarrow \infty$ as valid for a distant observer, the generalized Doppler factor in the emitter frame is evaluated to

$$
\begin{aligned}
g & =\frac{\alpha_{\mathrm{em}}}{\gamma\left[(1-\omega \lambda)-\alpha v^{(\mathrm{r})} \frac{\sqrt{\mathcal{R}_{0}}}{\rho \sqrt{\Delta}}-\alpha v^{(\theta)} \frac{\sqrt{\Theta}}{\rho}-\alpha v^{(\Phi)} \frac{\lambda}{\tilde{\omega}}\right]_{\mathrm{em}}} \\
& =\frac{\alpha_{\mathrm{em}}}{\gamma\left[1-\alpha v^{(\mathrm{r})} \frac{\sqrt{\mathcal{R}_{0}}}{\rho \sqrt{\Delta}}-\alpha v^{(\theta)} \frac{\sqrt{\Theta}}{\rho}-\lambda \Omega\right]_{\mathrm{em}}}
\end{aligned}
$$

Here, the already introduced Boyer-Lindquist functions are used again. Furthermore, there is the plasma velocity field in 
the ZAMO-frame (denoted by round brackets) $v^{(\mathrm{r})}, v^{(\theta)}$ and $v^{(\Phi)}$, two polynomials of the fourth order, $\mathcal{R}_{0}$ and $\Theta$, associated with the Carter Constant and the specific angular momentum $\lambda=J / E$, the ratio of the two conserved quantities along each photon path, angular momentum $J$ and total energy $E$. The polynomial $\mathcal{R}$ (in most general form, see Eq. (20)) becomes for photons $(m=0)$

$\frac{\mathcal{R}_{0}}{E^{2}}=r^{4}+\left(a^{2}-\lambda^{2}-C\right) r^{2}+2\left[C+(\lambda-a)^{2}\right] r-a^{2} C$.

Usually, one assumes Keplerian rotation for $r \geq r_{\mathrm{ms}}$ and sets

$v^{(\Phi)}=\tilde{\omega}\left(\frac{\Omega-\omega}{\alpha}\right)$.

The angular frequency, $\Omega$, has for radii greater than marginal stability a Keplerian profile

$\Omega=\Omega_{\mathrm{K}}= \pm \frac{\sqrt{M}}{\sqrt{r^{3}} \pm a \sqrt{M}}$ for $r>r_{\mathrm{ms}}$

(with upper sign for prograde, lower sign for retrograde orbits) and usually one forgets about the contributions of $v^{(\mathrm{r})}$ and $v^{(\theta)}$. These components can nevertheless be important for the emission line profile (Müller 2000). A more realistic and complicated velocity field than only Keplerian rotation will be discussed in the next section. For $r \leq r_{\mathrm{ms}}$, constant specific angular momentum is assumed. This is motivated by $1 \mathrm{D}$ hydrodynamic accretion disk models (Gracia 2002) and relativistic 2D hydrodynamic accretion disk models (Spindeldreher 2002). Here, one can set

$\Omega=\Omega_{\text {in }}=\omega+\frac{\alpha^{2}}{\tilde{\omega}^{2}} \frac{\lambda_{\mathrm{ms}}}{1-\omega \lambda_{\mathrm{ms}}}$ for $r \leq r_{\mathrm{ms}}$

with specific angular momentum at the marginally stable orbit given by

$\lambda_{\mathrm{ms}}=\frac{\tilde{\omega}_{\mathrm{ms}}^{2}\left(\Omega_{\mathrm{K}, \mathrm{ms}}-\omega_{\mathrm{ms}}\right)}{\alpha_{\mathrm{ms}}^{2}+\omega_{\mathrm{ms}} \tilde{\omega}_{\mathrm{ms}}^{2}\left(\Omega_{\mathrm{K}, \mathrm{ms}}-\omega_{\mathrm{ms}}\right)}$.

(The index ms refers to the quantity to be taken at the radius of marginal stability, $r_{\mathrm{ms}}$.).

In Fig. 5, the simulated distribution of the Doppler factor $g$ from Eq. (13) on a disk with parameters typical for Seyfert-1 galaxies $\left(i=30^{\circ}\right)$ is shown. First, one discovers the usual separation in blueshifted and redshifted segments due to rotation of the disk. The left-to-right symmetry is compared to the Newtonian case distorted. The left side of the disk rotates towards the observer and this radiation is beamed due to special relativistic effects: the matter rotation velocity becomes comparable to the speed of light. This fact can be derived from Fig. 6 , illustrating the prograde and retrograde velocity component, $v^{(\Phi)}$, in the ZAMO frame. The Keplerian profile of $v^{(\Phi)}$ holds only until reaching the radius of marginal stability, $r_{\mathrm{ms}}$. For smaller radii, one has to model this velocity component. The simplest way is to assume free-falling matter. In Fig. 6 the drift radius starts already at $R_{\mathrm{t}}=3.0 r_{\mathrm{g}}$. Then the test particle falls on geodesics onto the black hole. The velocity field is a complicated superposition of $v^{(\Phi)}$ and $v^{(\mathrm{r})}$. Besides, the plot depicts that

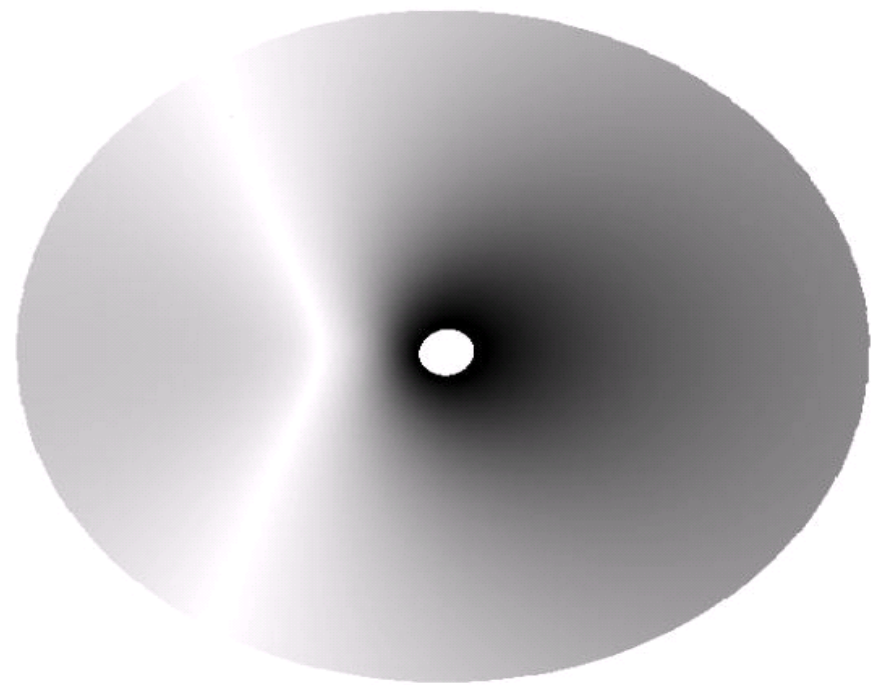

Fig. 5. Simulated disk image at inclination of $30^{\circ}$ and extreme Kerr $a=0.999999$ with color-coded distribution of generalized Doppler factor $g$ (white corresponds to $g=1$ ). The beamed blue approaching left side is clearly seen (triangular segment, $g>1$ ). Gravitational redshift (dark colors, $g<1$ ) darkens the inner edge until $g$ vanishes at the horizon.

the component $v^{(\Phi)}$ as observed by the ZAMO reaches a magnitude that is comparable to the speed of light: symmetrically in both cases, prograde and retrograde rotation, the maximum rotation velocity is about $0.4 \mathrm{c}$ ! Additionally, in both cases, at the horizon, this velocity component drops down to zero.

For completeness, the distribution of $g$ down to the event horizon of the black hole is calculated to illustrate mainly the effect of gravitational redshift. Certainly, a disk that touches the horizon is unphysical and would not be stable, unless in the case of maximum Kerr, $a=1.0$, where all characteristic black hole radii coincide to $1 r_{\mathrm{g}}$. Near the horizon the $g$-factor (Eq. (13)) significantly drops down and vanishes at the horizon itself (or equivalently, the redshift $z$ becomes infinity): the curvature of the black hole grasps everything that is near, even light. Figure 7 now shows the Doppler factor to the fourth power. In our approach, this is an essential ingredient for any emission originating from the vicinity of a black hole (ray tracing using transfer functions has a weight to the third power after all). This weight to the fourth power shows the relevance of the generalized Doppler factor. The $g$-factor significantly decreases inwards and becomes zero at the event horizon. The $g$-factor to the fourth power shows therefore a dramatic tininess around the black hole. This feature is called the central shadow of the black hole (Falcke et al. 2000). Likewise, one remarks a very bright region on the approaching part of the disk (beaming). For midly and highly inclined disks there is a chance to measure this clear brightness step, e.g. with sub-mm VLBI in the Galactic Center. This may be a method to prove the existence of the supermassive black hole with $3.7 \times 10^{6} M_{\odot}$ (Schödel et al. 2002) associated with the source Sgr A* by its radio emission. In near future, direct imaging of black holes may be possible! 


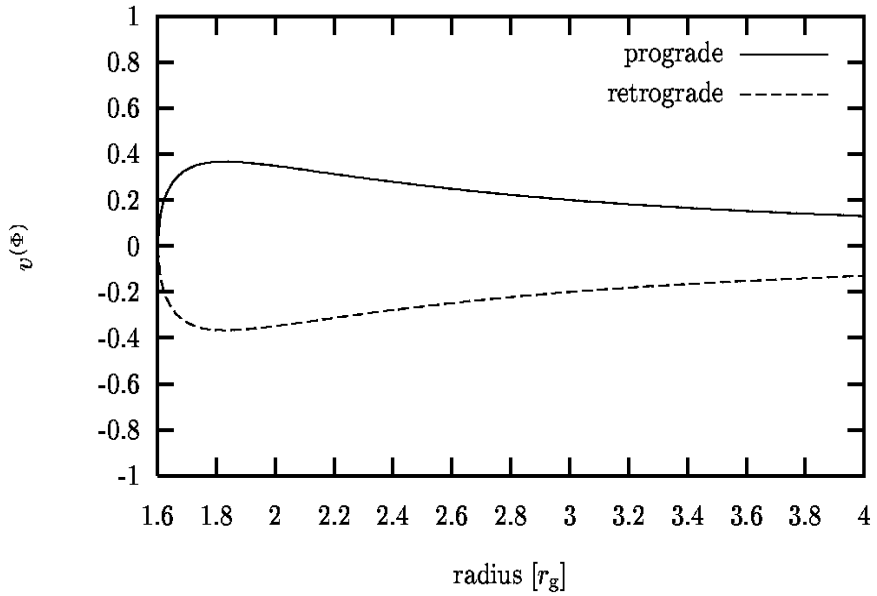

Fig. 6. Radial dependence of the velocity component $v^{(\Phi)}$ in the equatorial plane for Kerr parameter $a=0.8$. Prograde case is shown in the upper curve; retrograde case is illustrated in the lower curve. The matter starts in free-fall from a drift radius of $R_{\mathrm{t}}=3.0 r_{\mathrm{g}} \cdot v^{(\Phi)}$ vanishes self-consistently (for ZAMOs) at the horizon at $r_{\mathrm{H}}=1.6 r_{\mathrm{g}}$.

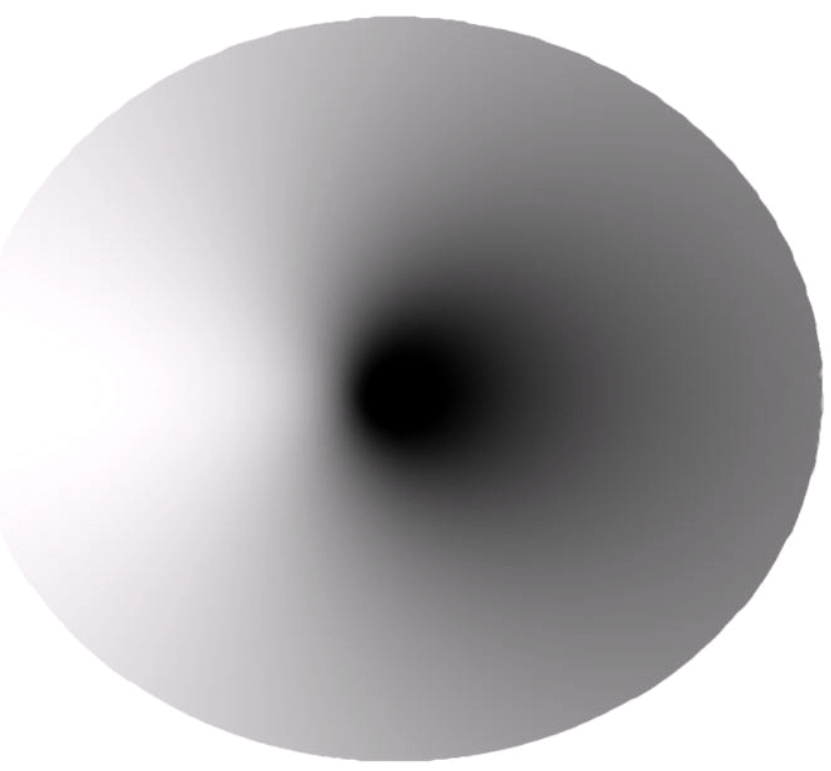

Fig. 7. Distribution of the Doppler factor $g^{4}$ over the disk. The inner disk edge touches at $r_{\text {in }}=1.0015 r_{\mathrm{g}}$ the horizon of the black hole with $a=0.999999$. The inclination angle is $30^{\circ}$ and the outer edge is at $r_{\text {out }}=30.0 r_{\mathrm{g}}$. The shadow of the black hole is clearly seen. Beaming on the left approaching side brightens any emission originating there. The right receeding part of the disk is darkened. This distribution is always folded in the flux integral and therefore important for any emission near black holes.

\section{The velocity field of the emitters}

The generalized Doppler factor $g$ depends on the plasma motions (here written in the ZAMO frame), $g=g\left(v^{(\mathrm{r})}, v^{(\Theta)}, v^{(\Phi)}\right)$. The usual issue forgets about the radial and poloidal components and derives line profiles from emitting plasma with only Keplerian motion, i.e. pure rotation. Recent non-radiative 3D-MHD pseudo-Newtonian simulations (Balbus \& Hawley 2002) again confirm this inflow and, additionally, the poloidal motion of disk winds. Therefore a more complicated plasma kinematics near the event horizon of Kerr black holes is investigated in this section. The behaviour of the velocity components $v^{(\mathrm{r})}, v^{(\Theta)}$ and $v^{(\Phi)}$ at the horizon is presented in detail below.

The radial velocity component will be determined by the accretion process. In this paper, it is considered that the radial drift starts at a certain radius, the truncation radius $R_{\mathrm{t}}$, that can even be greater than the radius for marginal stability $r_{\mathrm{ms}}$. Truncation is an essential feature of radiative accretion theory. This is motivated by radiative hydrodynamical accretion models of Hujeirat \& Camenzind (2000), discussed in Sect. 2. The correct free-fall behaviour in the Kerr geometry starting at radius $R_{\mathrm{t}}$ can be studied by introducing the radial velocity component

$v^{\mathrm{r}}=\frac{\mathrm{d} r}{\mathrm{~d} t}=\frac{\mathrm{d} r}{\mathrm{~d} \tau} \frac{\mathrm{d} \tau}{\mathrm{d} t}=\frac{\sqrt{\mathcal{R}}}{\rho^{2}} \frac{\alpha^{2}}{1-\omega \lambda}=\frac{\Delta}{\Sigma^{2}} \frac{\sqrt{\mathcal{R}}}{1-\omega \lambda}$,

where $\mathcal{R}$ denotes the radial potential (Carter 1968). This polynomial generally depends on Carter's constant $C$, specific angular momentum $\lambda$, total energy $E$ and mass of the plasma test particle $m$,

$\frac{\mathcal{R}}{E^{2}}=\left[\left(r^{2}+a^{2}\right)-a \lambda\right]^{2}-\Delta\left[\frac{C}{E^{2}}+(\lambda-a)^{2}+r^{2} \frac{m^{2}}{E^{2}}\right]$.

Carter's constant $C$ vanishes in the equatorial plane.

$v^{\mathrm{r}}$ is in a normalized ZAMO frame (indicated by round brackets)

$v^{(\mathrm{r})}=\frac{\exp \left(\mu_{\mathrm{r}}\right)}{\alpha}=\frac{\Sigma}{\Delta} v^{\mathrm{r}}$.

This quantity has to be evaluated in the equatorial plane, $\theta=\frac{\pi}{2}$. The specific angular momentum, $\lambda=J / E$, follows from

$$
\begin{aligned}
& \frac{E}{m}=\frac{r^{2}-2 M r \pm a \sqrt{M r}}{r \sqrt{r^{2}-3 M r \pm 2 a \sqrt{M r}}}, \\
& \frac{J}{m}= \pm \sqrt{M r} \frac{r^{2} \mp 2 a \sqrt{M r}+a^{2}}{r \sqrt{r^{2}-3 M r \pm 2 a \sqrt{M r}}},
\end{aligned}
$$

(upper sign prograde, lower sign retrograde orbits).

This provides the radial drift

$v^{(\mathrm{r})}=\frac{\sqrt{\mathcal{R}}}{\Sigma(1-\omega \lambda)}$

The kinematics at the event horizon,

$r_{\mathrm{H}}=M+\sqrt{M^{2}-a^{2}}$,

can be explored by evaluating the Boyer-Lindquist functions

$$
\begin{array}{cl}
\Delta\left(r_{\mathrm{H}}\right)=0, \quad \alpha\left(r_{\mathrm{H}}\right)=0, & \\
\Sigma_{\pi / 2}\left(r_{\mathrm{H}}\right)=2 r_{\mathrm{H}}, \quad \tilde{\omega}_{\pi / 2}\left(r_{\mathrm{H}}\right)=2, \\
\omega\left(r_{\mathrm{H}}\right)=a / 2 r_{\mathrm{H}} \equiv \Omega_{\mathrm{H}} .
\end{array}
$$

This finally yields

$$
\begin{aligned}
v^{(\mathrm{r})}\left(r_{\mathrm{H}}\right) & =\frac{1}{2 r_{\mathrm{H}}\left(E-\frac{a}{2 r_{\mathrm{H}}} J\right)}\left[E\left(1+2 \sqrt{1-a^{2}}+1\right)-a J\right] \\
& =\frac{1}{2 r_{\mathrm{H}} E-a J}\left[2 E\left(1+\sqrt{1-a^{2}}\right)-a J\right] \\
& =1 .
\end{aligned}
$$




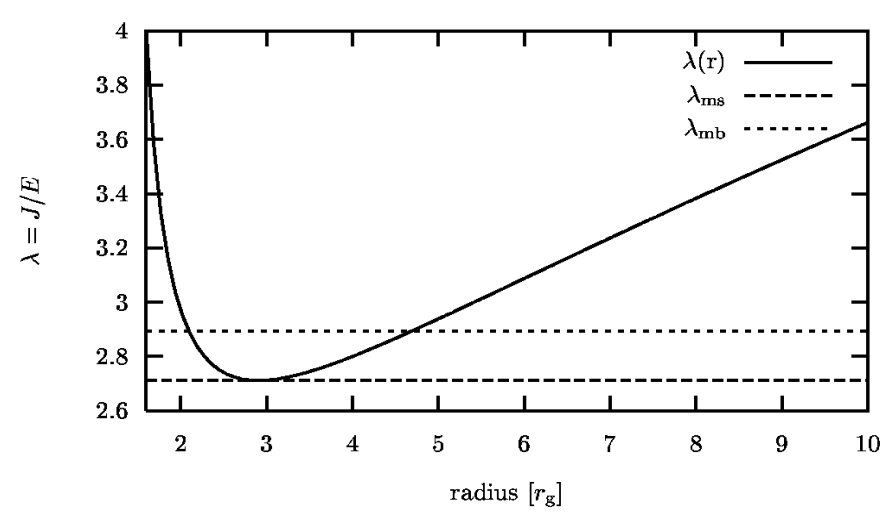

Fig. 8. Distribution of specific angular momentum, $\lambda(r)$, for Kerr parameter $a=0.8$. Fixing a constant specific angular momentum, $\lambda_{\mathrm{t}}$, is only allowed inbetween the two levels: the lower one, $\lambda_{\mathrm{ms}}$, and the upper one, $\lambda_{\mathrm{mb}}$.

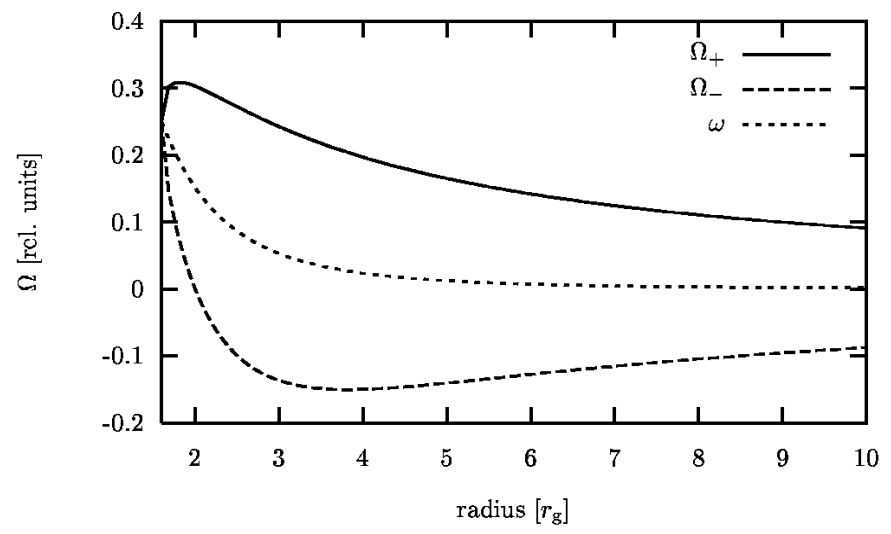

Fig. 9. Radial distribution of prograde (solid curve), $\Omega_{+}$, and retrograde (dashed curve) angular velocity limit, $\Omega_{-}$, for Kerr parameter $a=0.8$. The dotted curve illustrates the frame-dragging potential, $\omega$. At the horizon, here at $r_{\mathrm{H}}=1.6 r_{\mathrm{g}}$, all curves coincide. This is the visualization of the frame-dragging effect.

Therefore, matter in the equatorial plane enters the horizon with the speed of light. This is a general feature of the Kerr geometry and fully independent of any accretion model.

A realistic approach to model the radial drift is to fix the conservatives $E$ and $J$ when reaching the truncation radius $R_{\mathrm{t}}$ with the values that they take at this radius. Then, the particle will fall freely into the black hole. The disk is truncated at $R_{\mathrm{t}} \geq r_{\mathrm{ms}}$, the truncation radius gives the conservatives, $E\left(R_{\mathrm{t}}\right)$ and $J\left(R_{\mathrm{t}}\right)$. Otherwise, the radius of marginal stability fixes the conservatives to $E\left(r_{\mathrm{ms}}\right)$ and $J\left(r_{\mathrm{ms}}\right)$.

It is not possible to give arbitrary values for constant $\lambda_{\mathrm{t}}$, that means to fix arbitrary $R_{\mathrm{t}}$. As has been discussed in torus solutions on the Kerr geometry (Abramowicz et al. 1978), there is only an allowed interval of $\lambda_{\mathrm{ms}} \leq \lambda_{\mathrm{t}} \leq \lambda_{\mathrm{mb}}$ for the specific angular momentum, where ms denotes the orbit of marginal stability and $\mathrm{mb}$ the marginally bound orbit. This allowed interval $\lambda_{\mathrm{t}} \in\left[\lambda_{\mathrm{ms}}, \lambda_{\mathrm{mb}}\right]$ is depicted in Fig. 8 for a Kerr parameter of $a=0.8$. The general distribution of the specific angular momentum in the Kerr geometry is represented by the curve. The drift radius has to be chosen so that its according specific angular momentum, $\lambda_{\mathrm{t}}$, just lays inbetween the two horizontal lines. These lines belong to the specific angular momenta at the radius of marginal stability, $\lambda_{\mathrm{ms}}$, and at the marginally bound radius, $\lambda_{\mathrm{mb}}$. The physical reason for this restriction is, that $\Omega$ satisfies the condition

$\Omega_{-} \leq \Omega \leq \Omega_{+}, \Omega_{ \pm}=\omega \pm \frac{\alpha}{\tilde{\omega}}$.

These two limits are shown in Fig. 9 for $a=0.8$. It must be considered in modelling the radial drift with constant specific angular momentum that the resulting $\Omega$ lays inbetween these two curves, $\Omega_{+}$and $\Omega_{-}$. Otherwise, the model is invalid. This is because a global observer is globally time-like, $U_{\alpha} U^{\alpha} \leq 0$. By the frame-dragging effect, this observer becomes light-like, because the horizon is a light-like surface (null surface). If one chooses arbitrary specific angular momenta, one can run into trouble and injure the above allowed stripe for $\Omega$. In general, a slow rotating Kerr black hole, e.g. low Kerr parameter $a$, allows higher truncation radii. This can be investigated in Fig. 10 where models with different radial drift are compared. The models differ in Kerr parameter, $a$, and drift radius, $R_{\mathrm{t}}$, where the radial velocity component, $v^{(\mathrm{r})}$, starts to become finite. As can be seen, the radial velocity increases very steeply to smaller radii and becomes always the speed of light as has been proven analytically in Eq. (27). This component is the additive that changes the $g$-factor in Eq. (13) significantly. The essential consequence is that the gravitational redshift effect is enforced what can be directly investigated in Fig. 12: the inner edge shows a clear darkening due to smaller $g$-factors.

Let us now consider the toroidal velocity component, $v^{(\Phi)}$. From the Carter momenta it follows that

$v^{(\Phi)}=\frac{\tilde{\omega}}{\alpha}(\Omega-\omega)$.

Another general feature of the Kerr metric is the framedragging effect. Anything must co-rotate with the black holes horizon when reaching $r_{\mathrm{H}}$

$\Omega\left(r_{\mathrm{H}}\right) \equiv \Omega_{\mathrm{H}}=\omega$.

This can be immediately deduced from the general formula valid in the Kerr metric

$\Omega=\omega+\frac{\alpha^{2}}{\tilde{\omega}^{2}} \frac{\lambda}{1-\omega \lambda}$,

and the fact that $\alpha\left(r_{\mathrm{H}}\right)=0$. So, the azimuthal velocity in the ZAMO frame vanishes in general at the horizon as can be seen from Eq. (29). Figure 6 confirms this for $a=0.8$ and drift radius $R_{\mathrm{t}}=3.0$ The poloidal velocity component, $v^{\Theta}$, in the ZAMO frame

$v^{\Theta}=\frac{\mathrm{d} \theta}{\mathrm{d} t}=\frac{\mathrm{d} \theta}{\mathrm{d} \tau} \frac{\mathrm{d} \tau}{\mathrm{d} t}=\frac{\sqrt{\Theta}}{\rho^{2}} \frac{\alpha^{2}}{1-\omega \lambda}$

vanishes in the equatorial plane, hence the polynomial $\Theta$ defined by

$\frac{\Theta}{E^{2}}=\frac{C}{E^{2}}-\left[a^{2}\left(\frac{m^{2}}{E^{2}}-1\right)+\lambda^{2} \operatorname{cosec}^{2} \theta\right] \cos ^{2} \theta$

vanishes for $\theta=\pi / 2$. Therefore, the poloidal velocity component can be neglected for cold, infinitely thin standard disks. 


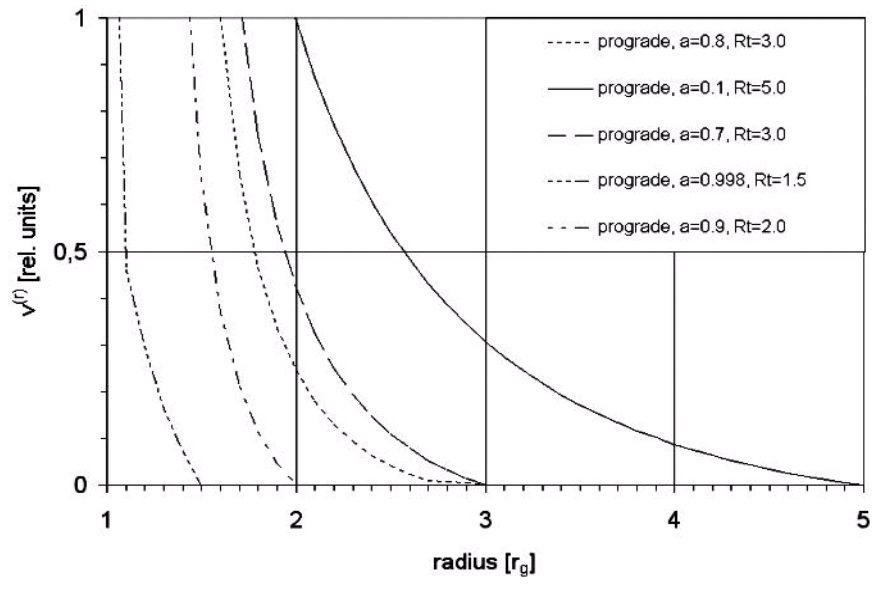

Fig. 10. Radial velocity component in the ZAMO for different radial drift models, that means different rotation state of black hole, $a$, and drift radius, $R_{\mathrm{t}}$. In all cases, the radial drift becomes light speed at the horizon radii.

\section{Radial emissivity profiles}

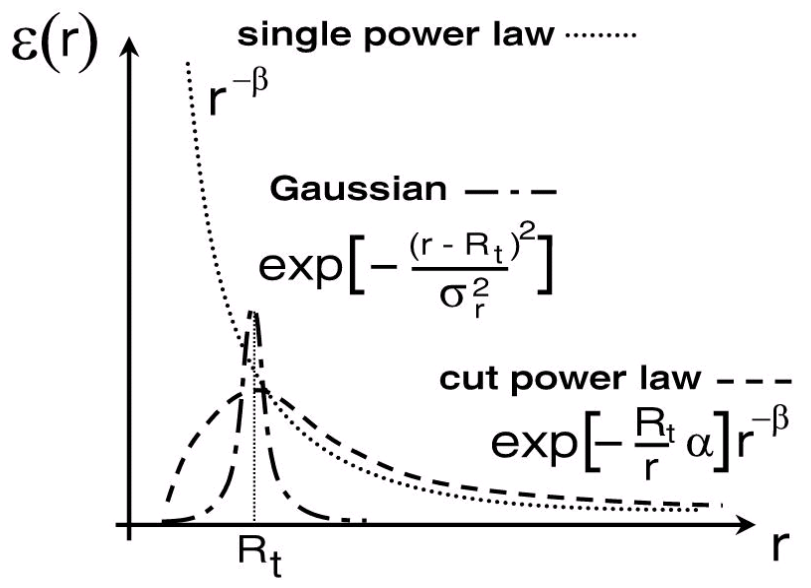

Fig. 11. Illustration of different radial emissivity models.

It may have relevance for slim or thick disks, when calculating their line flux. However this requires volume ray tracing, covariant radiation transfer and more physics as for standard disks what lies beyond the scope of this paper.

\section{Emissivity models}

In general, the disk emissivity is the quantity that has to be folded into the flux integral to evaluate the observed flux from accretion disks. One applies the emissivity as kind of profile function that has a radial and potentially an angular dependence. The resulting spectra (continuum flux, line flux) have therefore no physical flux units but arbitrary units.

The classical approach to model emissivities of accretion disks arose in the relativistic generalization of (nonrelativistic) SSDs (Page \& Thorne 1974; Novikov \& Thorne 1974). Here, the radial emissivity profile follows a single power law with slope index $\beta=3.0$

$\epsilon(r) \propto r^{-\beta}$.
This was motivated by the physics of standard accretion disks where other physical quantities follow this power law behaviour, too. Classical standard disks truncate at the radius of marginal stability, $r_{\mathrm{ms}}$, where consequently the emission of the disk breaks off naturally. In the historical issue (Page \& Thorne 1974) the Zero-torque boundary condition (ZTBC) has been applied. Then, the emissivity decreases steeply at this characteristic radius. Modern accretion theory resigns the ZTBC (Merloni \& Fabian 2003). On the one hand, this was theoretically motivated by efficient mechanisms to produce MHD turbulence, the well-known magneto-rotational instability (MRI, Balbus \& Hawley 1991). The MRI and the coupling of magnetized accretion disk to the black hole magnetosphere induces magnetically driven torques that injure the ZTBC. On the other hand, observations suggest significantly deviating disk emissivity profiles, $\epsilon(r) \propto r^{-4.5}$, giving a hint to higher dissipation at radii $r \leq r_{\mathrm{ms}}$ (Wilms et al. 2001).

The classical ansatz, $\beta=3$, was generalized later to a single power law index different from 3.0. The index $\beta$ may vary locally on the standard disk because different illumination from the hot corona and complex structure of the ionization layer on the disk can cause changes in the emissivity: $\beta$ becomes a fit parameter in $\mathrm{X}$-ray spectroscopy. Another aspect is, that at $r \gtrsim r_{\mathrm{ms}}$ the disk may be truncated. The inner accretion disk topology depends mainly on the total mass accretion rate as investigated in hydrodynamical accretion theory. For high accretion rate, the disk extends nearly to the black hole horizon and the ADAF (Narayan \& Yi 1994) is small. For low accretion rate, the ADAF is larger: a spheroidal evaporated hot region is attached to the standard disk and forms a sandwich configuration. A variable accretion rate is responsable for the different spectral states (very high, high, intermediate, low, quiescent) and radially oscillating transition radius between SSD and ADAF (Esin et al. 1997; Gracia 2002).

In the TSD scenario that emerges only in radiative accretion theory, it is possible that the matter then decouples from the standard disk in free-falling clouds. This plasma packages drift radially inwards and are advected from the black hole. This is major motivation of our new plasma velocity field model presented in Sect. 4. This phenomenon justifies an emissivity profile that does not decrease as sharp at radii comparable to $r_{\mathrm{ms}}$.

Furthermore, one can test so-called double power laws or broken power laws where at a certain radius the power law index jumps to another value.

In a simple approach, it is easy to modify the classical emissivity profile in the first step by a cut-power law. The most simple model would envisage a direct vertical cut in the emissivity profile. This may be over-simplified because it is expected that the emissivity is enhanced at the inner truncation due to stronger illumination by the corona. An even more adequate model considers the single power law with a modification by an exponential factor that suppresses the emission at smaller radii. Then, the emissivity profile decreases more smoothly at the truncation radius. Physically, this is motivated by pseudo-Newtonian, radiative two-component hydrodynamics accretion disk models in 2.5D axisymmetry (Hujeirat \& Camenzind 2000) where Comptonization, synchrotron radiation, bremsstrahlung and ion/electron conduction are 
considered. These simulations have shown that disk truncation at radii $r \gtrsim r_{\mathrm{ms}}$ seems to hold. It is supposed that this scenario is realized in AGN, miqroquasars and GBHCs.

Then, the radial emissivity in cut-power law form satisfies

$\epsilon(r) \propto \exp \left[-\frac{R_{\mathrm{t}}}{r} \alpha\right] r^{-\beta}$,

with truncation radius $R_{\mathrm{t}}$, steepness parameter $\alpha$ and single power law index $\beta$. The maximum of this distribution can be found at $r_{\max }=R_{\mathrm{t}} \alpha / \beta$. If one assumes a standard emissivity power law, $\beta=3.0$, for the second factor, one can fix $\alpha=3.0$, too. Then it is ensured that the emissivity peaks at $R_{\mathrm{t}}$ and exponentially decreases at smaller radii. This disk emissivity profile is comparable to the historical work (Page \& Thorne 1974) but exhibits a higher emissivity at the inner disk edge. This is plausible because $\mathrm{X}$-ray irradiation may be enhanced due to better illumination for geometrical reasons.

But there are alternatives to model the emissivity profile. In a new approach, a localized emissivity realized by a Gaussian emissivity profile is assumed

$\epsilon(r) \propto \exp \left[-\frac{\left(r-R_{\mathrm{t}}\right)^{2}}{\sigma_{\mathrm{r}}^{2}}\right]$.

One can easily control the width of the Gaussian by the parameter $\sigma_{\mathrm{r}}$. Narrow and broad Gaussians with constant $\sigma_{\mathrm{r}}$ are investigated. Another possibility is variable width by assuming $\sigma_{\mathrm{r}}=\eta R_{\mathrm{t}}$. Here the width scales with the truncation radius $R_{\mathrm{t}}, \eta$ denotes a constant factor that can be suitably chosen.

Gaussian emissivity profiles serve for modelling emission from a ring. In principle, the ring is the Greens function of the axisymmetric emission problem in the Kerr geometry. One can simply imagine that any spectral shapes originating from extended axisymmetric and flat emitters are composed of small rings, e.g. infinitely narrow Gaussians. Hence, one can produce a relativistic emission line profile by superimposing the flux data of several rings. Therefore, the Gaussian emissivity profile is well-suited to study different emitting regions.

Figure 11 illustrates all radial emissivities presented here in direct comparison.

\section{Visualization of standard disks}

With these preparations, the investigation of the emission distributed over the disk is straight-forward. One very interesting case is to study the influence of the radial drift $v^{(\mathrm{r})} \neq 0$, as presented in Sect. 4, Eq. (24). This new ansatz to model the velocity field results mainly in an enhancement of the gravitational redshift process (compare Fig. 12).

Another instructive example are high-inclined disks. Surely, this can not be applied to AGN type-1 and AGN type-2 are obscured by the large-scale dust torus, so that there is few chance to observe broad X-ray emission lines from type-2 AGN. But maybe this can be applied to stellar black holes in suitable orientation that can be found in microquasars and GBHCs. Besides, these studies are interesting to understand GR effects in this part of the parameter space.

A narrow ring laying directly around a Kerr black hole horizon is examined. Figure 13 now shows firstly the distribution of

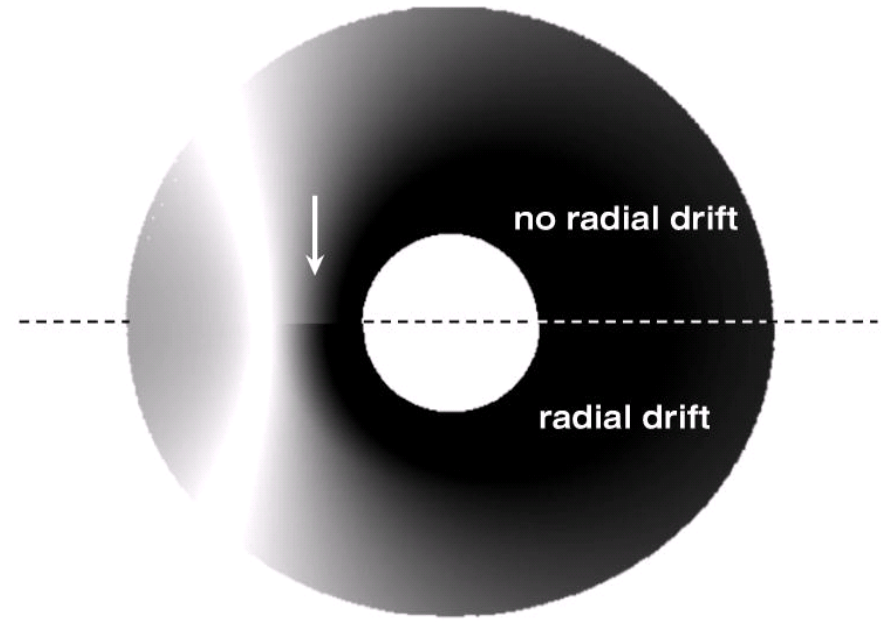

Fig. 12. Influence of radial drift. Upper hemisphere: pure Keplerian rotation $v^{(\Phi)} \neq 0, v^{(\mathrm{r})}=0$. Bottom hemisphere: Keplerian rotation plus radial drift $v^{(\Phi)} \neq 0, v^{(\mathrm{r})} \neq 0$. Enhanced gravitional redshift is clearly seen by darkening near the horizon indicated by an arrow. Parameters were $a=0.1, i=40^{\circ}, r_{\text {in }}=r_{\mathrm{H}}=1.996 r_{\mathrm{g}}, r_{\text {out }}=10.0 r_{\mathrm{g}}$. The radial drift starts at $R_{\mathrm{t}}=5.0 r_{\mathrm{g}}$.

the generalized Doppler factor $g$ from Eq. (13) under consideration of a Keplerian velocity component, $v^{(\Phi)}$, and a drift component, $v^{(\mathrm{r})}$ that is switched on at truncation radius $R_{\mathrm{t}}=3.0 r_{\mathrm{g}}$. Generally, for highly inclined disks the beaming of the approaching part is very strong. This illustrates the deep blue spot at the left disk segment in Fig. 13. The left approaching part of the disk exhibits a clear brightness step as can be investigated in the emission distributed over the disk. Apparently, this is a consequence of the $g$-factor jumping from high values in the beaming feature to very small values at gravitational redshift feature respective the horizon. If observers may find a possibility to look at high-inclined standard disks of cosmic black hole candidates, this step would be a distinct observable feature confirming the Kerr geometry ${ }^{2}$.

\section{Relativistic emission lines: Calculation and basic features}

Let us start with the calculation of the line flux from the rendered disk images. Traditionally in such calculations, it has been assumed that the emitting volumes are embedded into the surface of the disk and determine the generalized Doppler factor for each pixel in the image. So, in principle, one has to integrate over the disk images (that show the emission on each pixel), because a distant observer is not able to resolve the emitting disk. Physically, the observed spectral flux is evaluated generally by

$F^{\mathrm{obs}}=\int_{\text {image }} \mathrm{d} \Xi I_{v}^{\mathrm{obs}}$,

with the observed intensity $I_{v}^{\text {obs }}$ and an element of solid angle $\mathrm{d} \Xi$, covering a pixel of the disk as seen by a distant

${ }^{2}$ Color images of disk distributions and emission line profiles can be found at

http://www.lsw. uni-heidelberg.de/users/amueller 

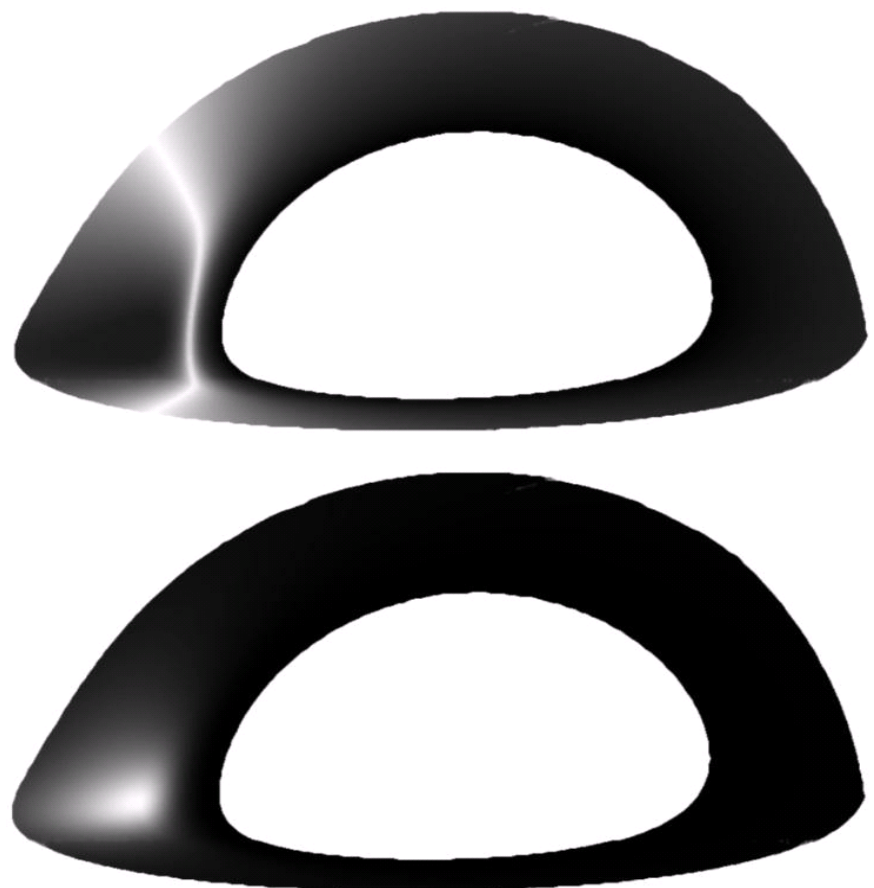

Fig. 13. The upper disk shows the distribution of the $g$-factor over a narrow ring with radial drift starting at $R_{\mathrm{t}}=3.0 r_{\mathrm{g}}$. White color indicates no net photon energy shift $(g=1)$, the left side is beamed $(g>1)$ whereas the right side and the regions near the horizon are strongly redshifted $(g<1)$. The inclination angle is $80^{\circ}$, the black hole rotation is fast, $a=0.8$, inner edge is the horizon at $r_{\mathrm{in}}=r_{\mathrm{H}}=1.6 r_{\mathrm{g}}$ and outer edge is only at $r_{\text {out }}=4.6 r_{\mathrm{g}}$. The lower disk indicates the associated distribution of the emission of the same narrow ring. White indicates high emission, black low emission. Here, the emissivity follows a broad Gaussian profile with $R_{\mathrm{t}}=3.0 r_{\mathrm{g}}$ and constant Gaussian width $\sigma_{\mathrm{r}}=3.0$.

observer. The observed intensity into the rest frame of the emitting plasma (denoted by^) holds

$I_{v}^{\mathrm{obs}}=g^{3} \hat{I}_{v}^{\mathrm{em}}$,

with the generalized Doppler factor, $g$, as introduced in Eq. (13). In the emitter frame, one has to make an ansatz for the intrinsic line shape. Usually, a Dirac delta function suffices to describe the transition at rest frame frequency $v_{0}$. One can assume a Gaussian profile motivated by Compton broadening, too. This is only one part, the frequency dependence. The other part is the radial disk emissivity that satisfies one of the models elaborated in Sect. 5. In our work it is consecutively assumed that

$\hat{F}_{v}^{\mathrm{em}}=\pi \hat{I}_{v}^{\mathrm{em}}=\epsilon(r) \delta\left(v_{\mathrm{em}}-v_{0}\right)$.

Finally, one has to consider a special feature of the delta distribution

$\delta\left(v_{\mathrm{em}}-v_{0}\right)=\delta\left(\left(v_{\mathrm{obs}}-g v_{0}\right) / g\right)=g \delta\left(v_{\mathrm{obs}}-g v_{0}\right)$.

So, plug all this into the flux integral (37) one gets:

$F_{\text {obs }}\left(E_{\text {obs }}\right)=\int_{\text {image }} \epsilon(r) g^{4} \delta\left(E_{\text {obs }}-g E_{0}\right) \mathrm{d} \Xi$, with observed flux, $F_{\text {obs }}$, line emission energy in the local rest frame, $E_{0}$, observed line energy in the observers frame, $E_{\mathrm{obs}}$, and solid angle element, $d \Xi$. Equation (41) shows that the flux is not a trivial integral over the disk but a complicated convolution of intrinsically emitted line weighted with the generalized Doppler factor to the fourth power and the radial emissivity function.

Line profiles are simulated that depend in general on the parameter set $\left\{a, i, r_{\mathrm{in}}, r_{\mathrm{out}}, v^{(\Phi)}, v^{(\mathrm{r})}, v^{(\Theta)}, \epsilon(r)\right\}$, with the Kerr parameter $a$, the inclination angle of the disk $i$, the inner $r_{\text {in }}$ and outer edge $r_{\text {out }}$ of the thin standard disk, the plasma velocity field in the ZAMO frame $v^{(\Phi)}, v^{(\mathrm{r})}, v^{(\Theta)}$ as elaborated in Sect. 4 and the emissivity law $\epsilon(r)$ that can depend on one, two or three parameters itself, as has been shown in Sect. 5.

Therefore, one deals with a rather huge parameter space that results in a variety of emission line shapes. Consideration of astro-chemistry makes it even more difficult, because different species and transitions ( $\mathrm{Fe} \mathrm{K} \alpha, \mathrm{Fe} \mathrm{K} \beta, \mathrm{Ni} \mathrm{K} \alpha, \mathrm{Cr} \mathrm{K} \alpha$ etc.) can contribute to the observed $\mathrm{X}$-ray line feature at approximately $6.5 \mathrm{keV}$.

Generally, there are three effects (Fabian et al. 1989) that influence the emission line profile ${ }^{3}$ : the Doppler effect, already known from classical Newtonian emitting disks, forms a characteristic double horn structure at medium to high inclinations. At low inclinations, the profile is triangular. The Doppler feature is for relativistic accretion disks distorted.

Beaming is a special relativistic effect. The rotating matter moves relativistically fast and the radiation is collimated (beamed) in direction of motion. Beaming intensifies the blue wing of emission lines with respect to the red wing. This effect is extraordinary important for disks with inner edges close to the event horizon because there the toroidal velocity as seen by ZAMOs is comparable to the speed of light. At the horizon itself, the toroidal velocity steeply decreases to become zero at $r_{\mathrm{H}}$ as shown in Fig. 6.

Gravitational redshift is a general relativistic effect. Matter and radiation near the black hole feels the strong curvature of space-time and looses energy when escaping this highlycurved region. The consequence is a shift of all photons that succeed in escaping the black holes sphere of action to the red branch of the spectrum. This effect results in an elongated red tail of the line. Besides, it lowers the relic red Doppler peak with respect to the blue wing.

The frame-dragging effect in the Kerr space-time forces anything to co-rotate. In particular, at the rotating horizon anything co-rotates with the angular velocity $\Omega_{\mathrm{H}}=\omega$ dictated by the black hole, compare Eq. (30). This means, that photons that do not rotate in the black holes direction may be forced to turn back. In Schwarzschild space-times, $a=0$, rotating matter is forced to stop rotation at the horizon, building up a boundary layer.

The combination of all these effects deform the spectral emission line to a very skewed profile with typically a long red tail (gravitational redshift), an intense beamed blue peak (beaming) and a double-peaked structure (Doppler boosting) as illustrated in Fig. 14. But the topology of the line depends

\footnotetext{
3 Thermal broadening has a tiny contribution and can be neglected.
} 


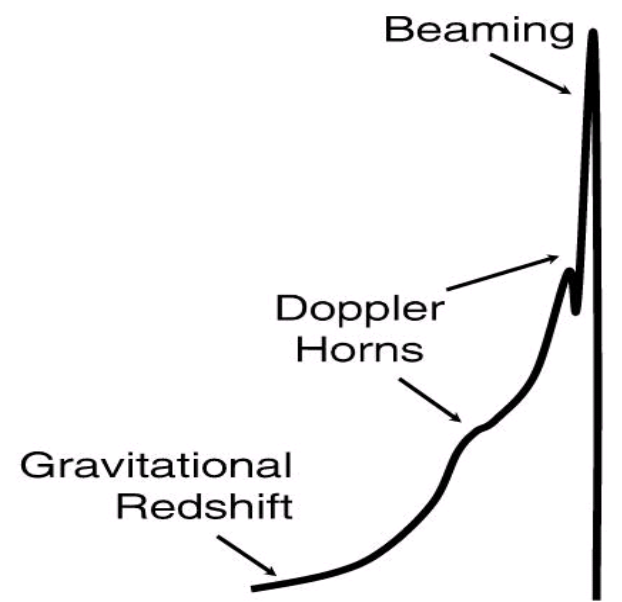

Fig. 14. Three effects form the line profile. This simulated profile of an iron $\mathrm{K}$ line has typical parameters for Seyfert-1 s. The observer is intermediately inclined, the plasma rotates only Keplerian and typical disk sizes $r_{\text {in }}=r_{\mathrm{ms}}, r_{\text {out }}=50 r_{\mathrm{g}}$ were taken.

strongly on the inclination angle and the emissivity law (see Sect. 5).

The parameters used to fit an relativistic emission line profile are numerous. In general, the strongest influence on both, broadness and topology, has the inclination of the disk. At low inclinations, the observer does not feel anything of the rotation of the disk and stares onto a nearly face-on oriented disk. There is no significant velocity component towards the observer. The emission line profile is triangular. This changes dramatically at high inclinations: the observer sees the approaching and receeding part of the rotating accretion disk. One can easily fix the inclination in a first approximation by determining the high-energy cut-off at the blue wing of the observed line. The emission line profile is double-horned.

Another aspect is the rotation of the black hole. For physical reasons, it seems clear that the Schwarzschild solution is ruled out. From stellar evolution theory it is known that stellar black holes formed by gravitational collapse of a rotating progenitor stars. Angular momentum can not radiated away by emission of gravitational waves. Therefore, at least stellar black holes are supposed to rotate. Even though the formation process of supermassive black holes is still enigmatic, one can assume that they form by merging of stellar Kerr black holes (hierarchical growth) or other rotating constituents. Then, it is very likely that black holes in general carry angular momentum. And even if black holes do not rotate from the beginning of their formation, the accretion mechanism makes sure that the Kerr solution is the approbiate metric: matter carries angular momentum and winds up the black hole spin when accreted (compare Sect. 3).

But the precise rotation state is unsure: it is not clear whether to take a Kerr parameter of 0.5 or 0.998 . It may be a good approach to assume high rotation near extreme Kerr $(a \approx 0.99)$ because these "elder black holes" accrete already for a long time and are wind-up strongly. Besides, the relativistic iron lines suggest that the emission originates from the innermost regions of Seyfert galaxies. A Kerr parameter of $a=0.5$ is associated with an orbit of marginal stability of $4.23 r_{\mathrm{g}}$. This may be to far away to cause the measured gravitational redshift effects. Disk truncation softens this argument as elaborated in Sect. 2.

As can be seen in the equation of the generalized Doppler factor (13), the velocity field of the radiation emitting matter is very important for the resulting emission line profiles. Typically, Keplerian motion (see Eq. (16)) has been taken into account (Fabian et al. 2000). Here, only the component $v^{(\Phi)}$ is non-vanishing whereas $v^{(\Theta)}$ and $v^{(\mathrm{r})}$ are zero. But it is certainly essential to incorporate a radial drift, e.g. a free-fall motion that is comparable to the modulus of the Keplerian motion when reaching the orbit of marginal stability. Here the correct fully relativistic free-fall formula in the Kerr geometry, Eq. (24), is adapted.

Poloidal components may also contribute. There is strong evidence that the jets of AGN and the "blobs" of GBHCs are magnetically driven and form in the direct neighborhood of the black holes horizon. The interaction of the (ergospheric) accretion disk with the black hole magnetosphere drives torsional Alfvén waves and finally an outflow (wind) that is collimated on larger scales to form jets. Certainly, the jets of Seyferts are weak and this argument holds rather for another type of AGN, the Quasars. Besides, to adequately consider poloidal motion, the ray tracer has to be modified to a volume ray tracer that renders the emission of $3 \mathrm{D}$ objects. But this has to be coupled to radiative MHD on the Kerr geometry, which is still an unresolved problem.

Recently, the variable iron line profiles from pseudoNewtonian MHD accretion theory have been calculated (Armitage \& Reynolds 2003). This model mimics the Schwarzschild geometry and shows nicely the dependence of the line flux from accretion but does still not incorporate relativistic effects of a Kerr black hole. This issue hints into the right direction of emission line simulations: coupling of accretion theory and X-ray spectroscopy. Another progress has been done to simulate non-radiative accretion flows on the Kerr geometry (De Villiers \& Hawley 2003). The next step will be to connect Relativity and radiation transfer to study radiatively cooled accretion flows on the background of the Kerr geometry. Here, in our first investigation, poloidal velocity components of the plasma are neglected. Firstly, the influence of radial drift and alternative emissivity models are studied.

One further important aspect is the disk size, especially the inner edge. The outer edge is rather irrelevant for classical emissivities, $\beta \geq 2.0$ (Reynolds \& Nowak 2003). The inner and outer radius of the disk are fixed and afterwards the image of this disk is determined. The historical approach is based on the famous SSD model and considers a thin and cold standard disk that extends outwards to several hundreds gravitational radii $\left(\sim 10^{2} r_{\mathrm{g}}\right)$ and inwards to the marginally stable orbit, $r_{\mathrm{ms}}$. For a rapidly rotating black hole $(a=0.998), r_{\mathrm{ms}}=1.23 r_{\mathrm{g}}$ is very close to the horizon $r_{\mathrm{H}}=1.063 r_{\mathrm{g}}$ ! Then gravitational redshift influences strongly the radiation from the inner disk edge. The accretion disk is supposed to be not as close to the black hole. This is motivated by pseudo-Newtonian radiative hydrodynamics simulations that show for typical accretion rates of Seyfert galaxies, $\dot{M}=0.01 M_{\odot} / \mathrm{yr}$, that the disk is truncated 
at $r \geq r_{\mathrm{ms}}$. Typical truncation radii $R_{\mathrm{t}}$ are 10 to $15 r_{\mathrm{g}}$, depending on the Kerr parameter $a$ and the allowed stripe introduced in Figs. 8 and 9.

\section{Line zoo: Topological classification and parameter space}

In this section, all types of line topologies emerging in our simulations are presented. From the plain line form, one can derive the following nomenclature of relativistic emission lines

$$
\begin{aligned}
& \text { - triangular, } \\
& \text { - double-horned, } \\
& \text { - double-peaked, } \\
& \text { - bumpy, } \\
& \text { - shoulder-like. }
\end{aligned}
$$

Figure 15 illustrates all prototypes of this terminology. As can be seen from the parameters, the triangular form follows from low inclination angles at rather high emissivity. This is trivial, because the Doppler effect is suppressed for nearly face-on observed disks: the red peak of the line vanishes.

Double-horned line shapes are somewhat standard profiles, because many astrophysical objects exhibit these typical form. Everything needed for that is a Keplerian velocity field, intermediate inclination, $i \approx 30^{\circ}$, and a standard single power law to reproduce a line profile with two Doppler boosted horns, where the blue one is beamed as usual.

The double-peaked profiles are well-known from the Newtonian case of higher-inclined radiating disks. Here, the width of the relic Doppler peaks is lower as compared to double-horned profiles. Mainly, this characteristic shape is a consequence of the space-time that is sufficiently flat, so that the typical red tail from gravitational redshift lacks. This can be theoretically reproduced by shifting the inner edge of the disk outwards. The simulations with stepwise shifting show nicely the "motion" of the red tail until it decreases as sharp as the blue edge. A relatively flat space-time is already reached around $25.0 r_{\mathrm{g}}$ corresponding to $0.25 \mathrm{AU}$ for a typical Seyfert galaxy with $10^{6} M_{\odot}$. Weakly accreting Seyfert galaxies with distant inner disk edges may fit this scenario.

Bumpy profiles are mainly caused by steep emissivity profiles. These emissivities cut away the emission from outer disk regions, especially the beaming segment on the disk as depicted in Fig. 5. Therefore, the line profile lacks the characteristic sharp blue beaming peak. The recent observation of Seyfert galaxy MCG-6-30-15 in the low state (Wilms et al. 2001) serves as an example of bumpy shapes.

Shoulder-like profiles exhibit a typically curved red wing. This feature is very sensitive to the parameters chosen. Our simulations showed, that only relatively narrow Gaussian, broken or cut-emissivities could produce such a distinct feature. The motivation to produce red shoulders arose from another observation of the Seyfert-1 galaxy MCG-6-30-15 (Fabian et al. 2002), now in the high state, showing such an extraordinary line shape. Blue shoulders can be produced by significant outflows, e.g. non-vanishing poloidal plasma velocity components (Müller 2000). Small shoulder-like features attached to the blue wing are typically interpreted as other species different from $\mathrm{Fe} \mathrm{K} \alpha$ such as $\mathrm{Fe} \mathrm{K} \beta$ (Fabian et al. 2002) or Ni K $\alpha$ (Wang et al. 1999). These transitions have rest frame energies above $7 \mathrm{keV}$.

Frequently, X-ray astronomers observe narrow but apparently separated lines that are superimposed to broad line profiles. The interpretation often given is that the two components originate from largely separated region: the broad line forms in the vicinity of the black hole whereas the narrow one is a reflection of $\mathrm{X}$-rays (coming from the central engine) at the large dust torus located on the kpc scale in AGN. Indeed, the dust harbors the relevant species $\mathrm{Fe}, \mathrm{Ni}$, etc. Therefore, these features should not be observed at microquasars (and stellar black holes in general) in default of a dust torus configuration. In simulations, it is possible to produce these narrow peak composites by adding an additional narrow Gaussian line profile without relativistic broadening.

After this topological classification, one can start to analyze the line characteristics numerically. Figure 16 illustrates a few line criteria that can be proposed to fix the line shape features. The utilisation of these criteria may simplify the comparison of observed and theoretically derived emission line profiles. Taking the absissa as a guideline, the minimum energy of the line, $E_{\min }$, the energy of the red relic Doppler peak (if visible), $E_{\mathrm{rp}}$, the energy of the blue relic Doppler peak (if visible), $E_{\mathrm{bp}}$, and finally the maximum energy of the line, $E_{\mathrm{max}}$, are introduced. The two Doppler peaks may serve to determine their energetic distance, the Doppler Peak Spacing:

$\mathrm{DPS}=E_{\mathrm{bp}}-E_{\mathrm{rp}}$,

or more generally in units of the generalized Doppler factor

$\mathrm{DPS}=g_{\mathrm{bp}}-g_{\mathrm{rp}}$.

On the spectral flux axis, $F_{\text {rp }}$ fixes the relic red and $F_{\mathrm{bp}}$ the relic blue Doppler peak. To infer an unit-independent quantity, one can calculate the flux ratio of these two and get the Doppler Peak Ratio:

$\mathrm{DPR}=F_{\mathrm{bp}} / F_{\mathrm{rp}}$

Additionally, the line reaches a maximum flux, $F_{\max }$, that may often match $F_{\mathrm{bp}}$ due to the beaming effect.

Another essential criterion that is even accessible by observation is the line width, in principle the total area of the emission line weighted by the energy where the line peaks, $E_{0}$. In many cases, $E_{0}$ may coincide with $E_{\mathrm{bp}}$ because beaming determines the maximum flux at the blue peak. The line width is evaluated numerically

$\mathrm{LW}=\frac{1}{E_{0}} \sum_{i} F_{\mathrm{i}} \Delta E$,

where $\Delta E$ is equal to the numerical spectral resolution of the emission line. More generally, one can express this in units of the $g$-factor to be independent of the line energy,

$\mathrm{LW}=\frac{1}{g_{0}} \sum_{i} F_{\mathrm{i}} \Delta g$, 

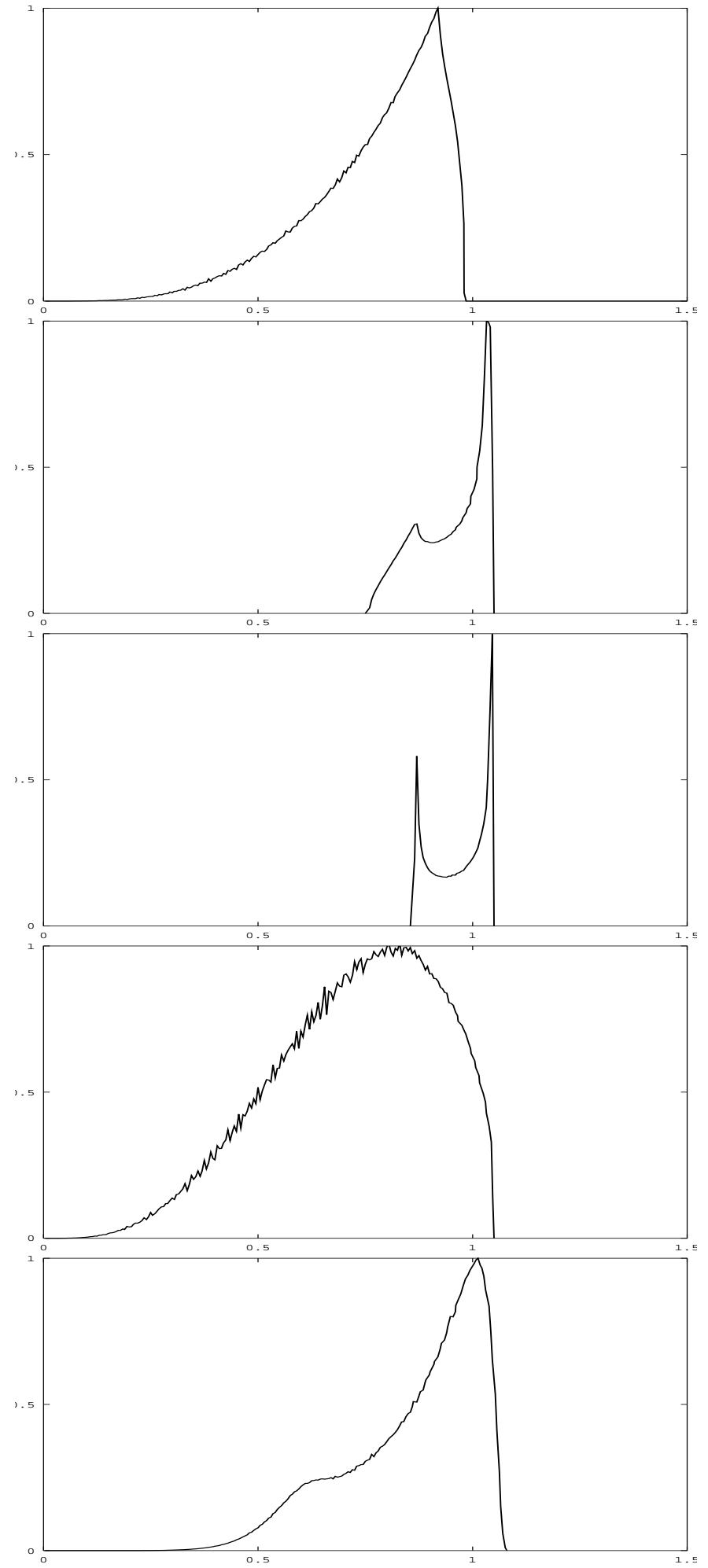

Fig. 15. A selection of topology types of relativistic emission lines. From top to bottom: triangular, double-horned, double-peaked, bumpy and shoulder-like. Line flux in normalized arbitrary units is plotted over $g$-factor.

with the corresponding $g$-factor $g_{0}=E_{0}^{\mathrm{obs}} / E_{0}^{\mathrm{em}}$. In both cases, constant spectral resolution $\Delta E_{\mathrm{i}}=\Delta E_{i+1}=\ldots=\Delta E$ respectively $\Delta g_{\mathrm{i}}=\Delta g_{i+1}=\ldots=\Delta g$ is assumed. Please consider that this line width has to be distinguished from the observationally accessible equivalent width.

Now, only a few selected parameter studies are presented because as mentioned before the parameter space is huge. The first phenomenon to explore is the frame-dragging effect or equivalently the depence of the line shape on the Kerr parameter $a$. Figure 18 shows only three lines at constant inclination angle, $i=40^{\circ}$, and classical emissivity power law, $\beta=3.0$, with variable Kerr parameter $a=0.999999$ (maximum Kerr), $a=0.5$ (intermediate case) and $a=0.1$ (close to Schwarzschild). The plasma kinematics is exclusively determined by Keplerian rotation. The inner disk edge is always coupled to the according horizon radius (Eq. (25)) and the outer edge is chosen so that the net area of the emitting disk is always constant. Then, the line profiles are compared and one can state that the flux grows with increasing $a$. This is because $r_{\text {in }}=r_{\mathrm{H}}$ decreases with increasing $a$ and the emissivity $\epsilon(r) \propto r^{-3.0}$ hence increases slightly extending to lower radii. This feature is therefore somewhat artificial, but may be justified by enhanced dissipation at the inner disk edge. In this case, the emissivity profile is supposed to contribute even for radii $r \leq r_{\mathrm{ms}}$. One can also state a changing line topology in this study: the ratios of the relic Doppler peak fluxes, $\mathrm{DPR}$, as introduced in Eq. (44), $\operatorname{DPR}(a=0.1) \approx 2.0$ and $\operatorname{DPR}(a=0.999999) \approx 1.7$, suggest that beaming is enhanced when the the black hole rotates faster. This is plausible by considering the increasing toroidal velocity, $v^{(\Phi)}$ respectively $\Omega$, with decreasing radius.

In Fig. 17 demonstrates line dampening by the shadow of the black hole. Here the radial drift model is applied, too, but a pure Keplerian velocity field does not change the following argument. The Gaussian emissivity shape provides a suitable tool to easily fix a small emitting ring laying around the horizon. The applied Gaussian emissivity peaks at $R_{\mathrm{t}}=1.5 r_{\mathrm{g}}$ and vanishes in principle for radii $r>2.0 r_{\mathrm{g}}$. Therefore, the line form is nearly symmetric. This examples depicts that even if line formation could happen within some $r_{\mathrm{g}} \lesssim r_{\mathrm{ms}}$, the line feature would be strongly suppressed by gravitational redshift. As analytically proven in Sect. 3 , the $g$-factor vanishes at the horizon. Therefore, the powers of $g$ (third or fourth, depending on ray tracing technique) folded into the flux integral reduce the observed flux. The consequence is a high-redshifted and damped emission line. As can be deduced from Fig. 17, a $\mathrm{Fe} \mathrm{K} \alpha$ line would peak at an observed energy of approximately only $3.4 \mathrm{keV}$. The line is significantly shifted to lower energies due to gravitational redshift. Obviously, it would be hard to observe this weak line. But in any case, the fluorescence process is supposed to be not possible in this proximity to the black hole because the accretion flow is too hot. The line dampening due to gravitational redshift is a strong competitive effect against models with steep emissivity single power laws as proposed in Wilms et al. (2001).

To demonstrate the influence of radial drift, a line set originating from a disk at three different inclination angles, $i=$ $10^{\circ}, 30^{\circ}, 60^{\circ}$, around a Schwarzschild black hole that extends from $r_{\text {in }}=r_{\mathrm{ms}}=6.0 r_{\mathrm{g}}$ to $r_{\text {out }}=30.0 r_{\mathrm{g}}$ with only Keplerian plasma motion is reproduced (Fabian et al. 2000). The same parameters are adapted but the inner disk edge is chosen to be at 


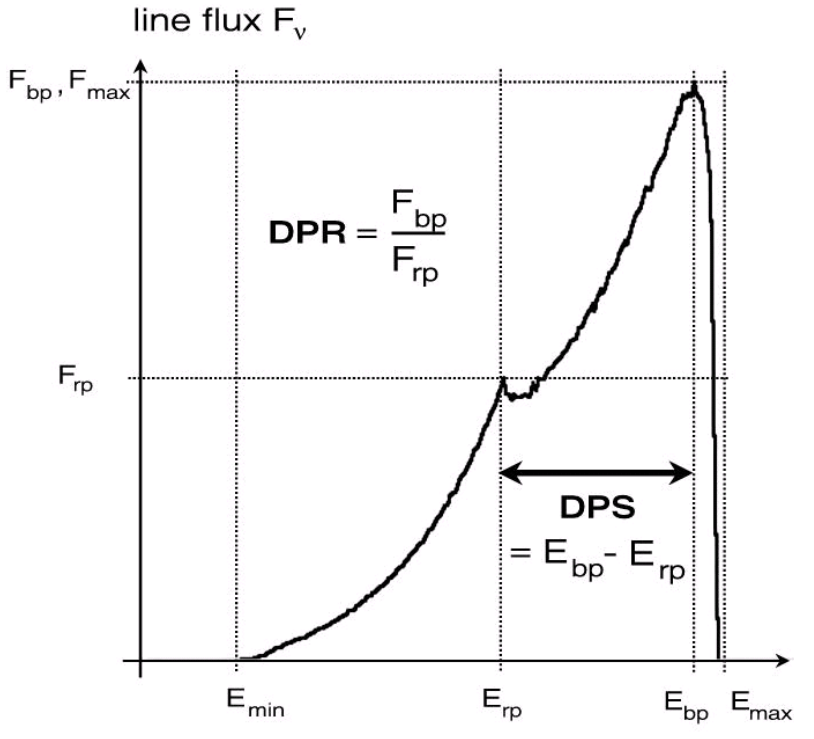

line energy $E$

Fig. 16. Illustration of line criteria to determine characteristics of a typical emission line profile. The position of the relic Doppler peaks (if existing) can be used to fix their flux ratio (Doppler Peak Ratio, DPR) and energetic distance (Doppler Peak Spacing, DPS). Other specific quantities are the maximal flux, $F_{\max }$, and the minimum and maximum energy of a line, $E_{\min }$ and $E_{\max }$. These criteria can be used to describe a line shape, observed or theoretically derived, with a few numerical values.

the horizon. Then, a component $v^{(\mathrm{r})} \neq 0$, e.g. a radial drift that starts at $R_{\mathrm{t}}=6.0 r_{\mathrm{g}}$ is considered. The emissivity law is chosen to a single power law with $\beta=3.0$ without inner cut-off to demonstrate the influence of emitting radially drifting plasma for $r \leq r_{\mathrm{ms}}$. Figure 19 depicts the result. A comparison of both line sets confirms that the blue edges remain almost constant. But radial drift starting already at the radius of marginal stability, $r_{\mathrm{ms}}=6.0 r_{\mathrm{g}}$ in Schwarzschild, suppresses the red relic Doppler peak by enhanced gravitational redshift effects. The red wings are in all three cases more smeared out with radial drift than in the case where only rotation is considered. For observation, this means that radial drift produces longer and more intense red tails of the emission line.

Now, the relativistic emission line profiles resulting from the new truncated accretion disk model presented here is investigated. The radial drift superimposed to the Keplerian motion as introduced in Sect. 4 and the new emissivity model with Gaussian shapes as has been shown in Sect. 5 are implemented. The missing link to this approach is the coupling of the Gaussian emissivity to the radial drift. This model can be justified by the following scenario: radiative accretion disks are truncated by efficient radiative cooling. This means that matter at the inner edge (at $R_{\mathrm{t}} \gtrsim r_{\mathrm{ms}}$ ) of the standard disk evaporates and matter clouds start to fall freely into the hole. This can be modelled with the radial velocity component $v^{(\mathrm{r})}$ as introduced in Eq. (24). At the horizon of the black hole, the plasma is mostly advected into the Kerr black hole and passes the horizon with the speed of light (analytically derived in Eq. (27) and illustrated in Fig. 10). The emissivity is modelled in a

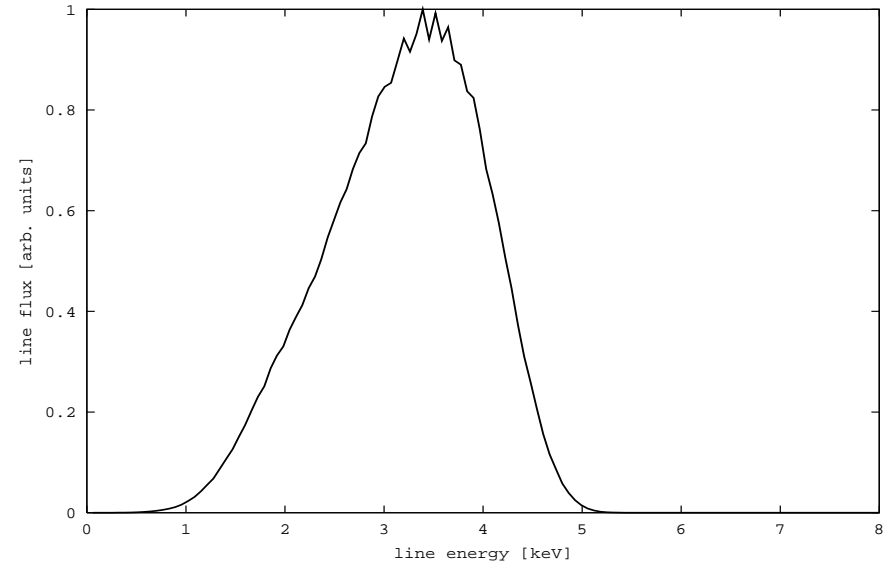

Fig. 17. Relativistic emission line forming directly in front of the horizon. The parameters were chosen to $i=30^{\circ}$, Kerr parameter $a=0.998$, disk size $r_{\text {in }}=r_{\mathrm{H}}=1.0633 r_{\mathrm{g}}, r_{\text {out }}=30.0 r_{\mathrm{g}}$, radial drift starting at $R_{\mathrm{t}}=1.5 r_{\mathrm{g}}$ and a localized emissivity by means of a Gaussian profile with $R_{\mathrm{t}}=1.5 r_{\mathrm{g}}$ and $\sigma_{\mathrm{r}}=0.4$. This more or less unphysical example for line emission should demonstrate the strongly suppressed emission due to the shadow of the black hole. Even if emission lines could form in the direct vicinity of black holes, the high power of the generalized Doppler factor $g$ reduces the flux and shifts the line significantly to lower energies due to gravitational redshift effects.

consistent manner: the illumination of the inner edge of the standard disk is expected to be increased because the disk exposes a larger surface to the primary X-ray source, e.g. a hot inner torus, an ADAF or generally speaking a corona. Therefore, the emissivity is higher than elsewhere on the accretion disk. This can be modelled by Gaussian emissivities that decrease very steeply to both wings of the emissivity shape. This is plausible, too, because at smaller radii there is only free-falling material that is optically thin and besides it is too hot to permit the fluorescence transition. At larger radii, the emissivity is expected to decrease, too, because the the surface of the disk becomes too cold for fluorescence. Hence, one can motivate to link the Gaussian profile of the emissivity to the drift radius. In a first step, broad Gaussians with constant width, $\sigma_{\mathrm{r}}=3.0$, are applied in all three cases depicted in Fig. 20. The black hole rotation state is constant, $a=0.1$. The drift radius varies from $R_{\mathrm{t}}=4.0,5.0,6.0 r_{\mathrm{g}}$ and is inserted in both, radial drift (Eq. (24)) and Gaussian emissivity (Eq. (36)). Figure 20 now shows that the line flux increases with increasing drift radius. The calculated line width, in principle the area below the line, even grows by a factor of 2 , if one compares $R_{\mathrm{t}}=4.0 r_{\mathrm{g}}$ with $R_{\mathrm{t}}=6.0 r_{\mathrm{g}}$. This is because the Gaussian width remains constant whereas the emitting ring (limited by Gaussian emissivity) grows in surface area. But a changing line topology can be derived, too. The red shoulder is elongated at $R_{\mathrm{t}}=4.0 r_{\mathrm{g}}$ because of enforced gravitational redshift for smaller drift radii respectively truncation closer to the black hole. Besides, the simulation yields a slight variation in the line form. This can be proven with the quantity DPR, the Doppler Peak Ratio which is independent from absolute flux values. This is the ratio of the flux from the blue Doppler peak versus the red Doppler peak. Surely, this can only be calculated if the two 


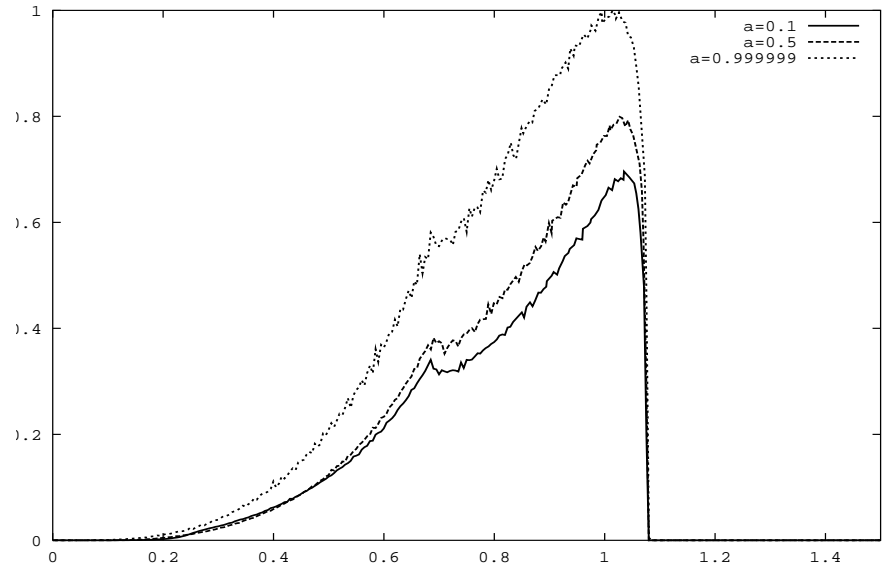

Fig. 18. Investigation of the dependence on the Kerr parameter $a$. The plasma motion is only Keplerian, the inclination is $40^{\circ}$. The black hole spin decreases from top to bottom line profile, $a=0.999999,0.5,0.1$. The inner disk edge is equal to the corresponding black hole horizon, $r_{\text {in }}=r_{\mathrm{H}}(a)$, and the disk edges are chosen so that the total disk surface remains constant.

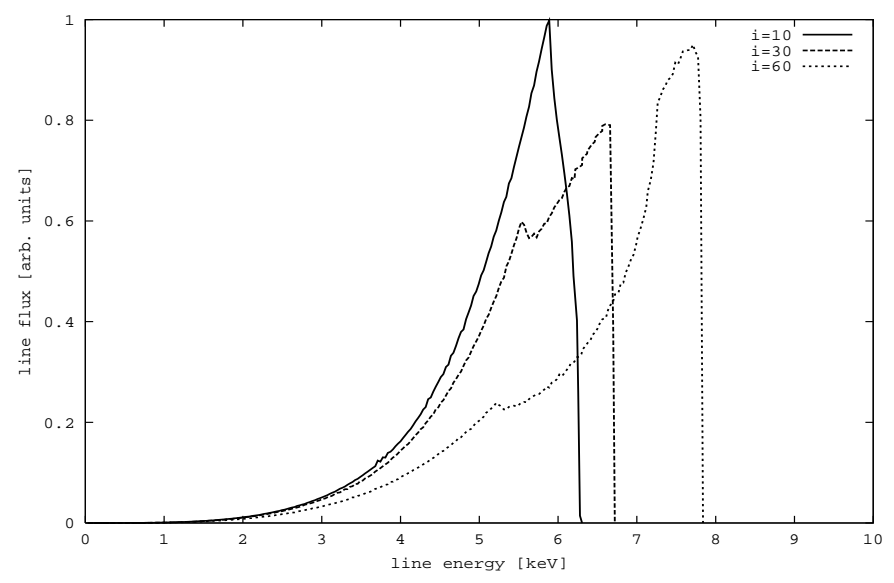

Fig. 19. Reproduction of the line set with variable inclination, $i=$ $10^{\circ}, 30^{\circ}, 60^{\circ}$ in Schwarzschild (Fabian et al. 2000). The main difference is that here an additional radial drift is adapted whereas Fabian et al. (2000) only assumed Keplerian motion. In both cases the emissivity follows a standard single power law. For better comparison, the abscissa is scaled to the iron $\mathrm{K} \alpha$ line with $6.4 \mathrm{keV}$ rest frame energy.

Doppler peaks exist. These DPRs are $\operatorname{DPR}\left(R_{\mathrm{t}}=4.0\right)=3.6$, $\operatorname{DPR}\left(R_{\mathrm{t}}=5.0\right)=4.0$ and $\operatorname{DPR}\left(R_{\mathrm{t}}=6.0\right)=4.3$. So, the red shoulder drops down with increasing $R_{\mathrm{t}}$ and the blue flux peak increases by the growth of the emitting region.

An alternative to the above issue, is to replace the Gaussian emissivity by the cut-emissivity profile as introduced in Eq. (35). This radial shape ressembles to the coronal emissivity profiles recently presented (Merloni \& Fabian 2003). For comparative reasons, all other parameters as in the example with Gaussian emissivity are maintained and only the emissivity is changed to a cut-power law with constant $\alpha=3.0$ and the same truncation radii set, $R_{\mathrm{t}}=4.0,5.0,6.0$. The behaviour at larger radii corresponds to the standard single power law $\propto r^{-3.0}$. The inferred emissivities are shown in Fig. 21. The resulting line profiles with these cut-emissivity shapes can be investigated in Fig. 22. For the three lines itself, the

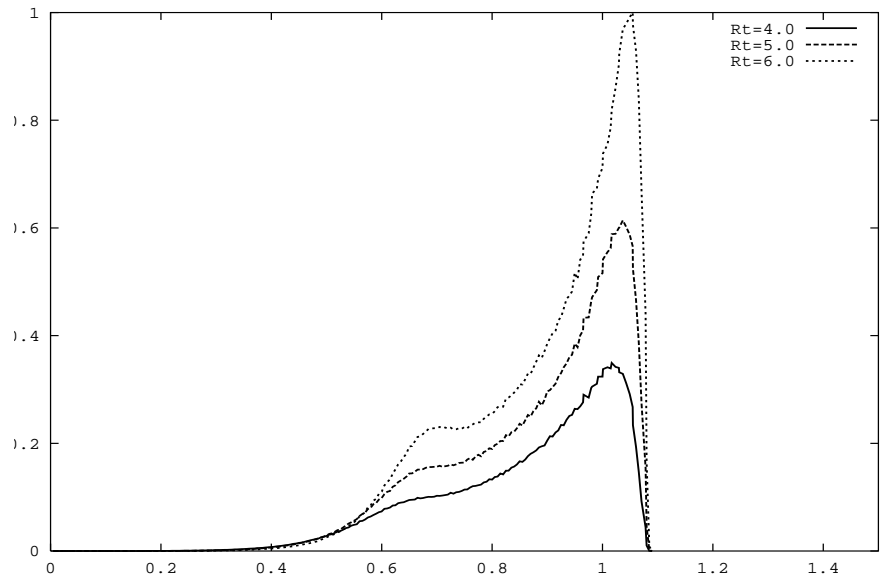

Fig. 20. Relativistic emission lines with radial drift model and Gaussian emissivity. The drift radius $R_{\mathrm{t}}$ from the plasma velocity field couples to the emissivity. From bottom to top, the drift radius increases, $R_{\mathrm{t}}=4.0,5.0,6.0$. The black hole rotates slowly, $a=0.1$, the inclination angle is $40^{\circ}, r_{\text {in }}=r_{\mathrm{H}}=1.995 r_{\mathrm{g}}$ and $r_{\text {out }}=30.0 r_{\mathrm{g}}$. The emissivity is modelled with constant $\sigma_{\mathrm{r}}=3.0$.

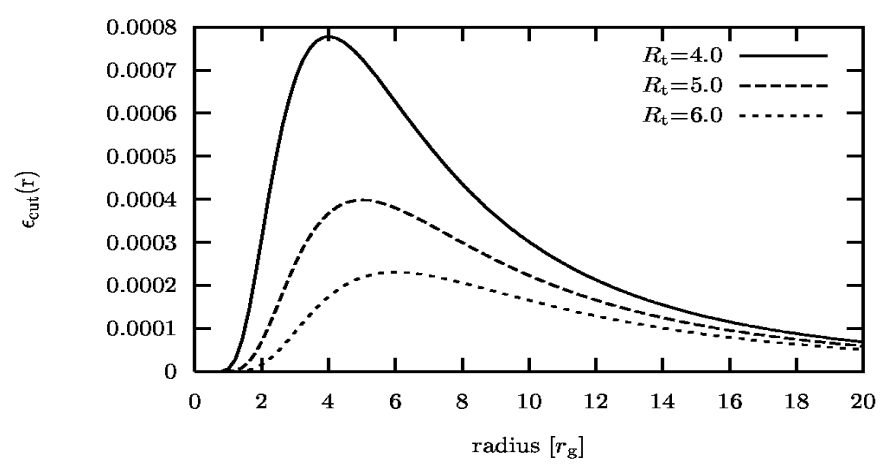

Fig. 21. Cut-emissivity with $\alpha=\beta=3.0$ for different truncation radii. From bottom to top, the drift radius increases, $R_{\mathrm{t}}=4.0,5.0,6.0$. With this choice of $\alpha$ and $\beta$, the emissivity peaks naturally at $R_{\mathrm{t}}$. For $r \leq R_{\mathrm{t}}$ the emissivity decreases exponentially.

allover topologies are almost identical, even DPS remains constant. But the ratio of the relic Doppler peaks slightly increases with $R_{\mathrm{t}}: \operatorname{DPR}\left(R_{\mathrm{t}}=4.0\right)=4.95, \operatorname{DPR}\left(R_{\mathrm{t}}=5.0\right)=5.16$ and $\operatorname{DPR}\left(R_{\mathrm{t}}=6.0\right)=5.26$. This effect is mainly caused by the fall of the red wing driven by the radial drift. Remarkable is the fact that the blue edge stays absolutely constant. Comparing Figs. 20 and 22, the blue edges remain constant. But, it is stressed that at the parameters chosen the Gaussian emissivity produces shoulder-like line shapes whereas the cut-power law emissivity produces double-horned profiles.

The reduction of the red wing flux caused by including radial drift is a generic feature. Figure 23 confirms this. Here two lines are compared where in one case a pure Keplerian velocity field applies and in the second case the radial drift model is used. This study reveals immediately the influence of including plasma drift. In this example, the drift starts at $R_{\mathrm{t}}=4.5$. One can see that the red part of the line drops down when drift is operating. The interpretation is that the gravitational redshift is enhanced by the drift, compare Eq. (13), and results in a lower red wing flux. This statement holds in principle for all 


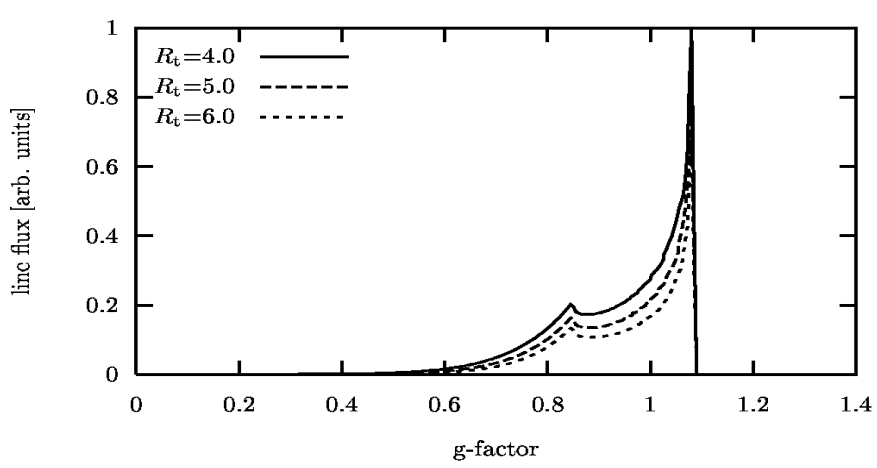

Fig. 22. Relativistic emission lines with radial drift model and cutpower law emissivity. The drift radius $R_{\mathrm{t}}$ from the plasma velocity field couples to the emissivity. From bottom to top, the drift radius increases, $R_{\mathrm{t}}=4.0,5.0,6.0$. The black hole rotates slowly, $a=0.1$, the inclination angle is $40^{\circ}, r_{\text {in }}=r_{\mathrm{H}}=1.995 r_{\mathrm{g}}$ and $r_{\text {out }}=30.0 r_{\mathrm{g}}$. The emissivity is modelled with constant $\alpha=\beta=3.0$.

simulated lines where the drift model is compared to its pure Keplerian counterpart.

Finally, a more complicated line profile with a few species in application to a recent observation of Seyfert-1 galaxy MCG-6-30-15 in the high state (Fabian et al. 2002) is demonstrated. The characteristic shoulder-like shape of this observation can be reproduced theoretically with the following plausible parameters:

- rapidly spinning black hole, $a=0.8$,

- typical Seyfert-1 inclination, $i=40^{\circ}$,

- inner edge at horizon, $r_{\text {in }}=r_{\mathrm{H}}=1.6 r_{\mathrm{g}}$,

- outer edge at $r_{\text {out }}=20.0 r_{\mathrm{g}}$,

- standard Keplerian rotation plus

- radial drift starting at $R_{\mathrm{t}}=4.5 r_{\mathrm{g}}$,

- Gaussian emissivity: $R_{\mathrm{t}}=4.5 r_{\mathrm{g}}$ and $\sigma_{\mathrm{r}}=0.4 R_{\mathrm{t}}$,

- multi-species line system consisting in $\mathrm{Fe} \mathrm{K} \alpha, \mathrm{Fe} \mathrm{K} \beta$, Ni $\mathrm{K} \alpha$ and $\mathrm{Cr} \mathrm{K} \alpha$.

The relative line strength of the different species are modelled in an approximation found in Reynolds (1996). Figure 24 now illustrates a line system consisting of iron, nickel and chromium. The total observed X-ray fluorescence feature around $7 \mathrm{keV}$ is equal to the sum of these contributions. The theoretically inferred line FWHM is very high, around $1 \mathrm{keV}$, which has the same order as the $\approx 750 \mathrm{eV}$ as can be found in Fabian et al. (2002). These parameters fit nicely the observed shoulder-like red wing and even the small blue bump beyond the $\mathrm{Fe} \mathrm{K} \alpha$ beaming branch. The flux ratio between shoulderlevel and maximal flux around a factor of 5 is close to the observed value of 4 . The other elements seem to be vital to model this observed profile. However, chromium seems to play a minor role due to weak relative strength.

\section{Conclusions}

It has been demonstrated that radial drift, a non-vanishing radial velocity component, is a vital ingredient in modelling relativistic emission line profiles. Accretion theory demands on such more complicated non-Keplerian velocity fields.

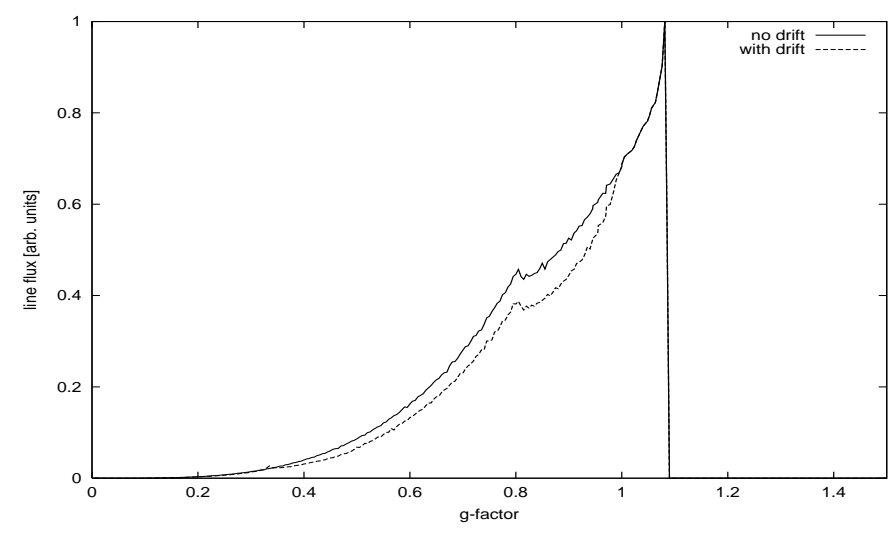

Fig. 23. Direct confrontation of relativistic emission lines with and without radial drift model. A single-power law emissivity with $\beta=$ 3.0 is chosen. The black hole spin parameter is $a=0.8$, the inclination angle is $40^{\circ}, r_{\text {in }}=r_{\mathrm{H}}=1.6 r_{\mathrm{g}}$ and $r_{\text {out }}=20.0 r_{\mathrm{g}}$. The pure Keplerian case, top line, is modelled by constant specific angular momentum for $r \leq r_{\mathrm{ms}}=2.91 r_{\mathrm{g}}$. The non-Keplerian case, bottom line, includes drift starting at $R_{\mathrm{t}}=4.5 r_{\mathrm{g}}$.

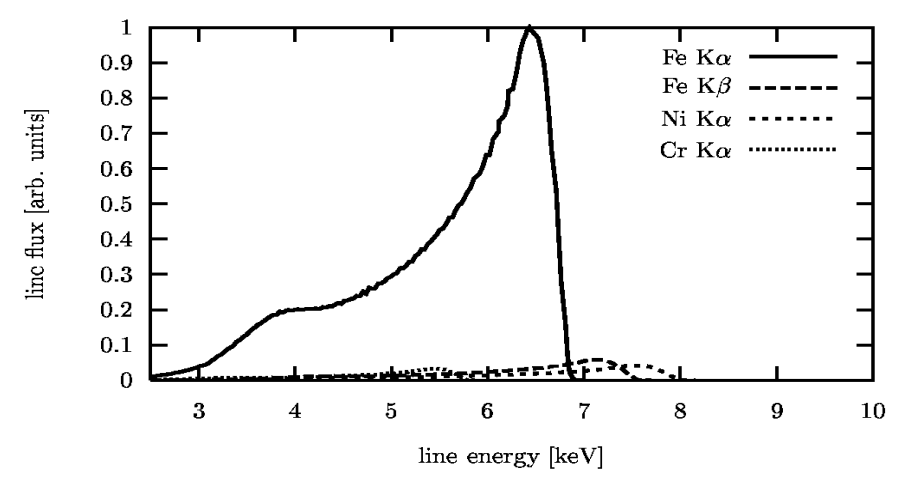

Fig. 24. Suggestion of a multi-species line system fitting the XMMNewton observation of MCG-6-30-15 (Fabian et al. 2002). A line complex consisting of $\mathrm{Fe} \mathrm{K} \alpha$ at $6.4 \mathrm{keV}, \mathrm{Fe} \mathrm{K} \beta$ at $7.06 \mathrm{keV}$, Ni K $\alpha$ at $7.48 \mathrm{keV}$ and one weak contribution of $\mathrm{Cr} \mathrm{K} \alpha$ at $5.41 \mathrm{keV}$ (all energies measured in the rest frame) is simulated.

However, our approach is just a phenomenological model motivated by simulations of accreting black hole systems. Some analytical features, as the asymptotical behaviour at the black hole horizon are independent of these models. One main consequence is, that the radial ZAMO velocity becomes the speed of light at the horizon whereas the toroidal ZAMO velocity vanishes there. Another essential feature in accreting and radiating black hole systems is the steep decrease of the generalized Doppler factor causing a shadow of the black hole. Hence, any emission near black holes is strongly suppressed. This is the competing dominant effect that suppresses as well steep emissivity profiles within the marginally stable orbit.

The truncated standard disk (TSD) hypothesis induced by radiative pseudo-Newtonian hydrodynamics is a new aspect in accretion theory that also influences the models for X-ray spectroscopy, especially the formation of broad relativistic emission lines. Future fully relativistic and radiative MHD is expected to confirm this. Then, a coupling of $3 \mathrm{D}$ ray tracing techniques and plasma dynamics are inevitable to explain the morphology and variablity of relativistic emission lines, particularly the iron 
$\mathrm{K} \alpha$ line. Besides, one hope is that these demanding simulations will enlighten the open question of the corona topology. The answer is essential to understand $\mathrm{X}$-ray irradiation of thin accretion disks.

Alternative disk emissivity models, such as cut-power laws and Gaussian emissivities can serve as valuable tools to study the huge parameter space of the line zoo. The localized emissivity studies with Gaussian emissivity laws prove that emission from regions neighboring the black hole horizon is massively suppressed by the low generalized Doppler factor. Additionally, the exponentially decreasing cut-power law may be a suitable profile function to model the disk emissivities with higher magnetically driven dissipation at the inner disk edge.

A first classification of the variety of relativistically broadened emission lines succeeds by a topological criterion: the lines can be divided in families with triangular, double-horned, double-peaked, bumpy and shoulder-like line form. It turns out that this first step constrains the parameter space significantly.

Unfortunately, it is still not feasible to solve the fully covariant radiation transport problem. Therefore, one deals always with some restrictions in the accretion models: either the approach is fully relativistic, but neglects radiation or the model incorporates radiation processes, but mimics relativity only to a certain degree by pseudo-Newtonian potentials. The future of accretion theory points towards radiative 3D-GRMHD. Until this will be possible, one has to extract few informations from "simpler" models with limitations done in the past (such as pseudo-Newtonian models, non-radiative issues, less dimensions etc.) and puzzle this input to an understanding of black hole accretion physics.

Acknowledgements. Part of this work was supported by the Deutsche Forschungsgemeinschaft, DFG (Sonderforschungsbereich 439).

\section{References}

Abramowicz, M., Jaroszynski, M., \& Sikora, M. 1978, A\&A, 63, 221 Armitage, P. J., \& Reynolds, C. S. 2003, MNRAS, submitted [astro-ph/0302271]

Balbus, S. A., \& Hawley, J. F. 1991, ApJ, 376, 214

Balbus, S. A., \& Hawley, J. F. 2002, ApJ, 573, 738

Ballantyne, D. R., Ross, R. R., \& Fabian, A. C. 2001, MNRAS, 327, 10

Blandford, R. D., \& Znajek, R. L. 1977, MNRAS, 179, 433

Bromley, B. C., Chen, K., \& Miller, W. A. 1997, ApJ, 475, 57

Carter, B. 1968, Phys. Rev., 174, 1559

Chandrasekhar, S. 1983, The Mathematical Theory of Black Holes (Oxford University Press)

Cunningham, C. T. 1975, ApJ, 202, 788

Dabrowski, Y., \& Lasenby, A. N. 2001, MNRAS, 321, 605

De Villiers, J. P., \& Hawley, J. F. 2003, ApJ, 592, 1060

Dewangan, G. C., Griffiths, R. E., \& Schurch, N. J. 2003, ApJ, 592, 52
Esin, A. A., McClintock, J. E., \& Narayan, R. 1997, ApJ, 489, 865

Fabian, A. C., Rees, M. J., Stella, L., \& White, N. E. 1989, MNRAS, 238, 729

Fabian, A. C. 1998, Astron. \& Geophys., 123

Fabian, A. C., Iwasawa, K., Reynolds, C. S., \& Young, A. J. 2000, PASP, 112, 1145

Fabian, A. C., Vaughan, S., Nandra, K., et al. 2002, MNRAS, 335, L1

Falcke, H., Melia, F., \& Agol, E. 2000, ApJ, 528, L13

Fanton, C., Calvani, M., Felice, F., \& de Cadez, A. 1997, PASJ, 49, 159

Gracia-Calvo, J. 2002, Time-dependent accretion flows onto black holes, Ph.D. Thesis, Landessternwarte Heidelberg

Gracia-Calvo, J. 2003, MNRAS, submitted [astro-ph/0301113]

Hujeirat, A., \& Camenzind, M. 2000, A\&A, 361, L53

Iwasawa, K., 1996, MNRAS, 282, 1038

Kerr, R. P. 1963, Phys. Rev. Lett., 11, 237

Lee, J. C., Ogle, P. M., Canizares, C. R., et al. 2001, ApJ, 554, L13

Manmoto, T., \& Kato, S. 2000, ApJ, 538, 295

Mason, K. O., Branduardi-Raymont, G., Ogle, P. M., et al. 2003, ApJ, 582,95

Matt, G., Fabian, A. C., \& Ross, R. R. 1993, MNRAS, 262, 179

Matt, G., Fabian, A. C., \& Reynolds, C. S. 1997, MNRAS, 281, 175

Merloni, A., \& Fabian, A. C. 2003, MNRAS, 342, 951

Müller, A. 2000, Emissionslinienprofile akkretierender Scheiben um rotierende Schwarze Löcher, diploma thesis, Landessternwarte Heidelberg

Narayan, R., \& Yi, I. 1994, ApJ, 428, L13

Novikov, I. D., \& Thorne, K. S. 1974, Black Holes, 343

Page, D. N., \& Thorne, K. S. 1974, ApJ, 499, 191

Penrose, R., \& Floyd, R. M. 1971, Nat. Phys. Sci., 229, 177

Pfefferkorn, F., Boller, T., \& Rafanelli, P. 2001, A\&A, 368, 797

Pounds, K. A., Nandra, K., Stewart, G. C., et al. 1990, Nature, 344, 132

Reeves, J. N., Turner, M. J. L., Pounds, K. A., et al. 2001, A\&A, 365, L134

Reynolds, C. S. 1996, X-ray emission and absorption in active galactic nuclei, Ph.D. Thesis, University of Cambridge

Reynolds, C. S., \& Nowak, M. A. 2003, Phys. Rep., 377, 389

Ross, R. R., Fabian, A. C., \& Young, A. J. 1999, MNRAS, 306, 461

Rybicki, G. B., \& Lightman, A. P. 1979, Radiative Processes in Astrophysics (New York: John Wiley \& Sons)

Schödel, R., Ott, T., Genzel, R., et al. 2002, Nature, 419, 694

Shakura, N. L., \& Sunyaev, R. A. 1973, A\&A, 24, 337

Speith, R., Riffert, H., \& Ruder, H. 1995, Comput. Phys. Commun., 88,109

Spindeldreher, S. 2002, The Discontinuous Galerkin Method applied on the equations of ideal relativistic hydrodynamics, Ph.D. Thesis, Landessternwarte Heidelberg

Tanaka, Y., Nandra, K., Fabian, A. C., et al. 1995, Nature, 375, 659

Turner, T. J., George, I. M., Nandra, K., \& Mushotzky, R. F. 1997, ApJ, 488, 164

Wang, J.-X., Zhou, Y.-Y., \& Wang, T. G. 1999, MNRAS, 328, L27

Wilms, J., Reynolds, C. S., Begelman, M. C., et al. 2001, MNRAS, 328, L27

Yaqoob, T., George, I. M., Nandra, K., et al. 1999, ApJ, 525, L9 